\title{
3Dino System, come accorciare le distanze nei rilievi di precisione
}

\author{
Sara Antinozzi \\ Diego Ronchi \\ Salvatore Barba
}

Abstract

|| rapido susseguirsi di innovazioni tecnologiche per la digitalizzazione del patrimonio, così come l'incertezza sulle condizioni e sulla continuità della sua fruizione - con evidente riferimento alla conservazione del bene, ma anche alle recenti condizioni di inaccessibilità -, impongono un approfondimento sulla strutturazione di procedure di rilievo tridimensionale e virtualizzazione. Queste, prevalentemente indirizzate a ordini di grandezza e scale di restituzione architettonica, sono però poco orientate verso la codifica e la formalizzazione di pratiche di rilievo per oggetti di piccolissime dimensioni; la questione è resa ancor più singolare dall'esigenza di acquisire geometrie e superfici altamente uniformi e/o riflettenti che, come noto, rendono più complesso - negli algoritmi di Structure from Motion - il riconoscimento dei punti omologhi.

In prove da noi condotte con i microscopi digitali portatili della Dino-Lite abbiamo testato le potenzialità dell'impiego di immagini 'micro' anche nel campo del Cultural Heritage, evidenziando, però, dei necessari sviluppi tecnologici, specialmente legati alla profondità di campo e alla conseguente geometria di presa.

Questo nuovo studio è teso a risolvere le problematiche e a incrementare le prestazioni della fotogrammetria microscopica, ottimizzando le procedure di acquisizione con la progettazione di accessori personalizzati per la micro-fotogrammetria. I risultati sono stati perseguiti nell'ambito di un accordo di trasferimento tecnologico finalizzato ad avanzare un protocollo per la documentazione fotogrammetrica di piccoli artefatti.

Parole chiave

microscopi portatili Dino-Lite, cultural heritage, small artefacts, monetazione antica, micro-fotogrammetria.

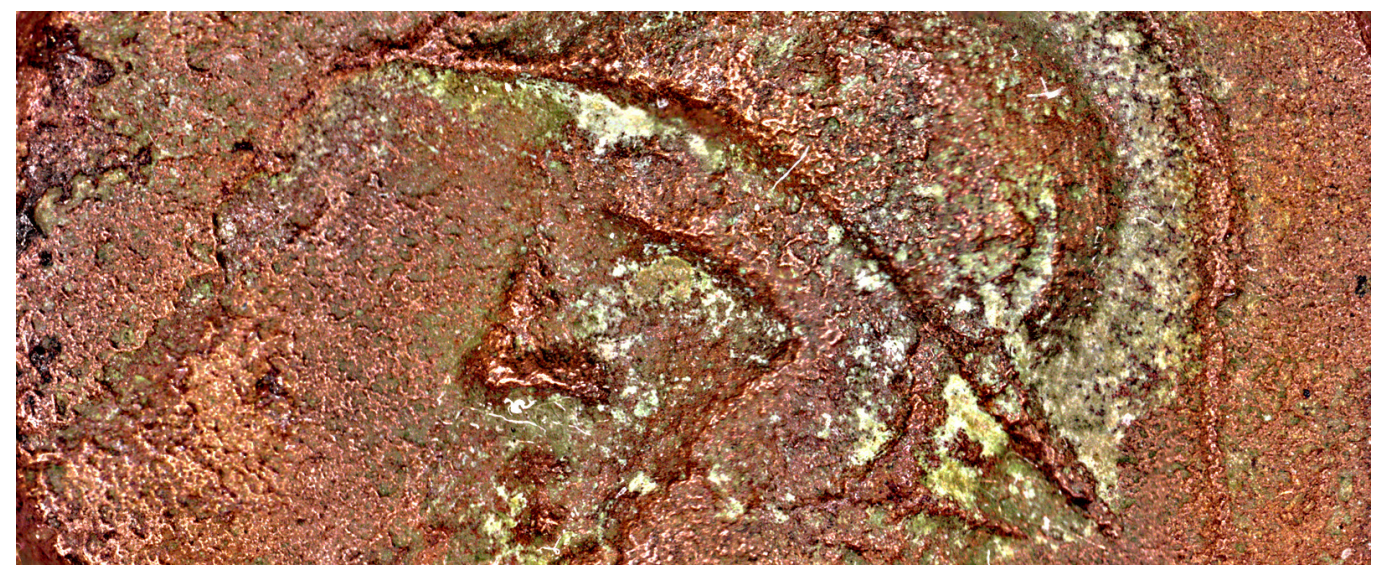




\section{Introduzione}

II continuo miglioramento di sensori, tecniche di acquisizione e sistemi computazionali costituisce la vera forza degli strumenti per la registrazione e visualizzazione dei dati [Adamopoulos, Rinaudo 2019, pp. I-6]. Ciò ha aperto la strada a processi sempre più automatizzati, con accuratezza crescente, caratterizzati da tempi e costi contenuti; circostanza che infittisce la rete di rapporti di complementarità e interazione tra le diverse discipline correlate all'imaging e all'elaborazione tridimensionale, tra cui l'industria manifatturiera, le scienze mediche, l'intrattenimento e, ovviamente, il patrimonio culturale [Pieraccini, Guidi, Atzeni 200 I, pp. 63-70]. L'incremento della domanda di misurazioni tridimensionali di oggetti con superfici complesse e caratteristiche morfologiche sub-millimetriche ha generato diverse soluzioni di tipo range-based per applicazioni micrometriche [Hansen et al. 2006, pp. 72 I -743]. Tuttavia, non di rado, le limitazioni sia ottiche che meccaniche e i costi non trascurabili indirizzano l'interesse verso una fotogrammetria di precisione, un paradigma rapido, accurato e più economico, confacente al rilievo di superfici piccole e articolate [Galantucci, Pesce, Lavecchia 20 I6, pp. 2 II-2 I9], anche se a discapito, spesso, della ricostruzione dell'intero volume del campione [Gontard et alt. 20 16, pp. 80-88]. A tale proposito, l'avvento di microscopi digitali portatili per l'ispezione, la documentazione e l'analisi digitale metrologica - già popolari nel settore industriale della produzione e del controllo di qualità, nonché impiegati anche in campo medico - ha svelato una potenzialità non trascurabile per la modellazione di piccoli oggetti, raggiungendo accuratezze dell'ordine del decimo di millimetro [Esmaeili, Ebadi 20 I7, pp. 65-67]. Nel giro di pochi anni, la possibilità di utilizzare fotografie scattate da un microscopio ha destato l'attenzione anche di settori meno affini a quello produttivo-industriale, come quello dei beni culturali; tra le motivazioni più cogenti, sussiste l'esigenza di rendere accessibile una grande mole di ritrovamenti, spesso di dimensioni molto contenute, giacente nei magazzini dei nostri musei, anche per finitezza degli spazi espositivi, esigenza a cui di recente si è sommata la chiusura alla fruizione in presenza. Prescindendo dall'urgenza di esporre e catalogare questi oggetti, l'impiego di rappresentazioni 3D digitali introdurrebbe miglioramenti significativi nel lavoro delle figure professionali correlate all'ambito del Cultural Heritage, inquadrando il bene in una prospettiva globale e olistica, inserendolo in un vasto sistema di conservazione delle informazioni, a un tempo modificabile e implementabile [Apollonio et al. 202 I, pp. I-40].

L'interfacciarsi con reperti di piccole dimensioni determina non solo un cambiamento di scala di rappresentazione, ma anche un ripensamento dei sistemi operativi di acquisizione, in contesti non sempre confortevoli [Plisson, Zotkina 2015, pp. I02-I I9]. L'impiego di mi-

Fig. I. II calibratore tridimensionale '3Dino sezion 'con il dettaglio della sezione di uno dei for
troncoconici.

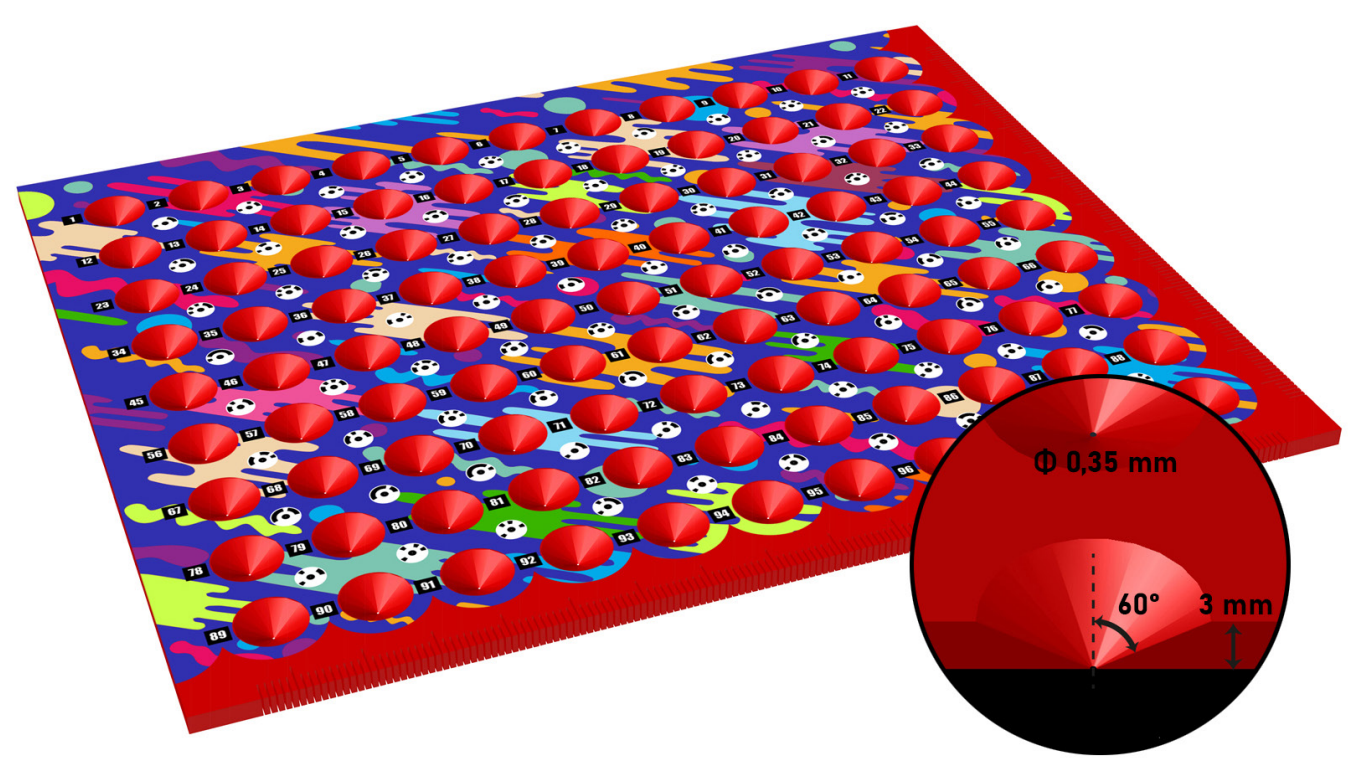


croscopi per fini fotogrammetrici, ostico a causa del campo visivo ristretto e della ridotta profondità di campo che ne caratterizzano il sistema ottico, rende - difatti - innovativa la sfida di adattare tali strumenti alle peculiarità del nostro settore scientifico disciplinare. Così, come in altre aree della fotogrammetria close-range, il vantaggio dell'elaborazione fotogrammetrica microscopica digitale risiederà, una volta assunto il pieno controllo di tutte le variabili di sistema, da un lato nella possibilità di codificare un'automazione del processo di acquisizione [Mitchell, Kniest 1999, pp. 695-704] e dall'altro nel perseguire accuratezze adeguate e verificabili.

Sono attualmente disponibili sul mercato strumentazioni range-based che soddisfano le esigenze mensorie illustrate, ma a costi non sempre contenuti [Tolksdorf, Elburg, Reuter 2017, pp. 400-4I0] e non così efficaci nel ri-posizionamento dei singoli pixel sulla superficie se comparati a sistemi image-based [Bolognesi, Fiorillo, 20 I8, pp. 337-342], o approcci integrati che rappresentano ancora la soluzione più efficace [Morena, Barba, Álvaro-Tordesillas 2019, pp. I 35- | 42]. || contributo, pertanto, si focalizzerà sulla sistematizzazione di strumenti hardware, singolarmente già disponibili, per esplorare la fotogrammetria microscopica al fine di definire una procedura standardizzata per l'acquisizione di oggetti di piccolissime dimensioni con dettagli e texture complesse.

\section{Caso studio: il rilievo di una moneta bronzea del I secolo a.C.}

Il rilievo di precisione di un'antica moneta di bronzo proveniente da Velia, antica polis della Magna Grecia oggi appartenente al Parco Archeologico di Paestum e Velia, ha rappresentato un'occasione, in un certo qual modo emblematica, per sperimentare le performance di alcuni microscopi digitali in ambito fotogrammetrico.

Gli interrogativi che gli studi di numismatica antica pongono, in particolar modo per l'esame dei coni, inducono a non fare affidamento unicamente su documentazione e analisi tradizionali, costituite prevalentemente da misurazioni manuali e fotografie; un tipo di documentazione che può risultare limitante in termini di acquisizione delle informazioni e per successivi approcci analitici. Metodi più avanzati per la registrazione e l'esame del reperto si stanno gradualmente affermando nelle pratiche di conservazione e in ambito museale, divenendo a oggi quasi la norma [Hess, MacDonald, Valach 2018, pp. I-22].

L'oggetto da noi selezionato, per stabilire se il processo di registrazione digitale proposto possa supportare i risultati della ricerca, è una moneta di circa $1,2 \mathrm{~cm}$ e del peso di 2,93 g (numero di inventario 223964, adatta per dimensioni e per la natura della sua superficie), che al dritto presenta una testa elmata di Atena volta a sinistra e al rovescio un tripode con le lettere 'YELH', quale riferimento al nome greco della città di Velia, dal nome della ninfa Yele. II reperto fa parte di un gruppo di oltre mille esemplari simili, riferibili alla stessa produzione, attualmente oggetto di studi [I] e databili alla seconda metà del I secolo a.C. Per giungere a una qualità descrittiva adeguata il rilievo è stato condotto con due differenti microscopi digitali portatili della Dino-Lite (<www.dino-lite.eu>), avvalendosi di una piastra calibrata - progettata dagli autori e realizzata per l'occasione - al fine di ottimizzare le procedure di allineamento e messa in scala del modello tridimensionale. L'intenzione, infatti, in questa fase della ricerca, è stata quella di incrementare il potenziale descrittivo di questi microscopi.

\section{Materiali e metodi: 3Dino Plate \& Dino-Lite handheld digital microscopes}

L'attività di ricerca con il sistema micro-fotogrammetrico 'battezzato' 3Dino si è basata, quindi, sull'impiego combinato di microscopi portatili a luce polarizzata della Dino-Lite e di un originale calibratore tridimensionale disegnato ad hoc. Quest'ultimo, il 3Dino Plate, è costituito da una piastra in PLA ottenuta con una stampa 3D a filamento, delle dimensioni di $18 \times 15 \mathrm{~cm}$ e caratterizzata da un pattern ortogonale di 99 fori troncoconici, con un angolo di svasatura di $60^{\circ}$ e diametro della base minore pari a 0,35 mm (fig. I). 


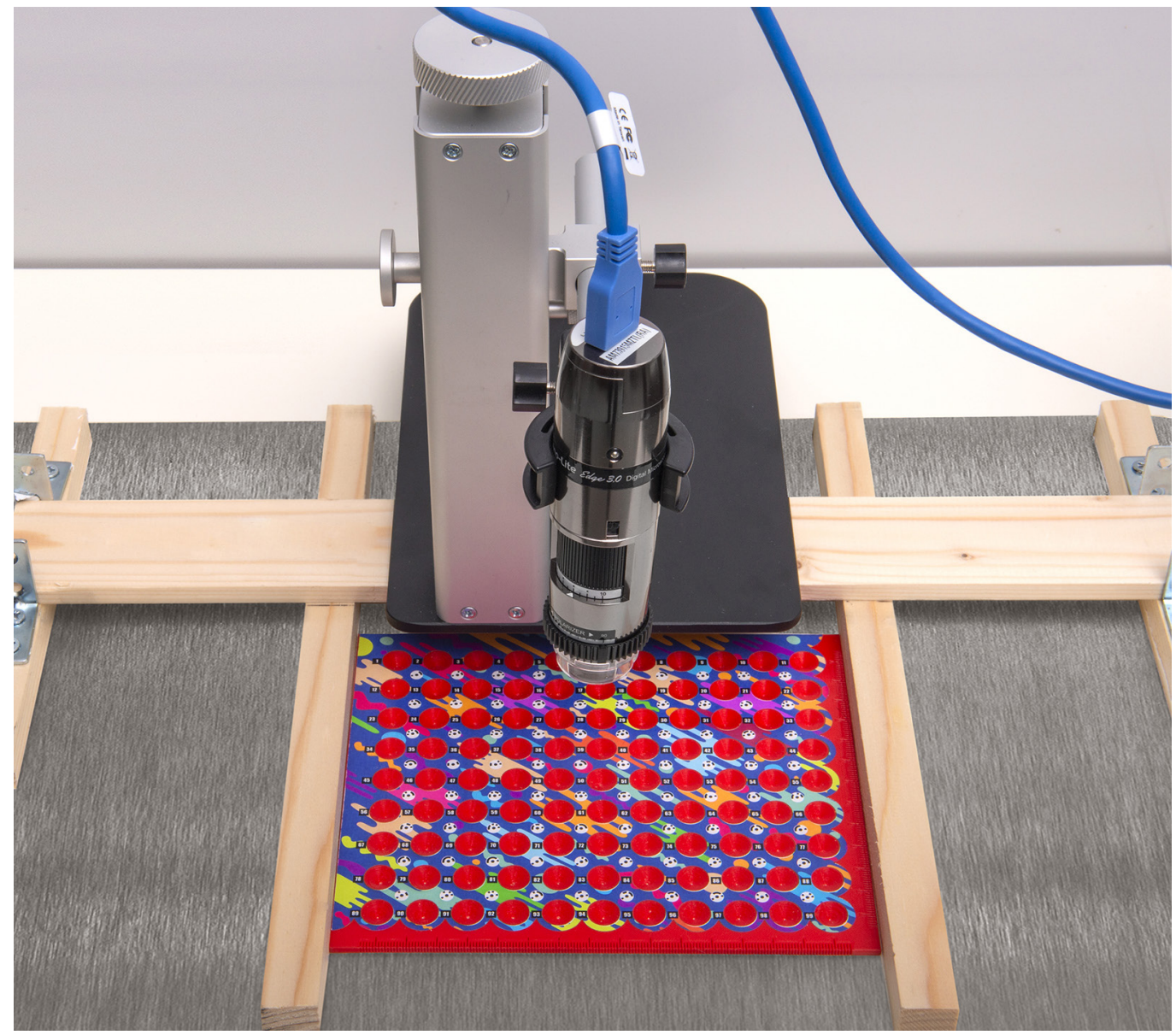

L'accuratezza del calibratore, in base ai settaggi [2] della stampa 3D e alle valutazioni dei relativi scarti, ovvero alla conformità della posizione effettiva dei fori rispetto al file di progetto, è stata stimata in $0,1 \mathrm{~mm}$, assumibile come valore dell'errore strumentale del sistema (ossia l'accuratezza dei markers).

Le coordinate di ciascun foro - rispetto a un sistema di riferimento locale - sono dunque note all'operatore e possono essere importate nel progetto fotogrammetrico: ciò consente di utilizzare il pattern forato come una griglia di punti di vincolo (GCP), omogeneamente distribuita su tutta l'area di interesse per l'ottimizzazione dei parametri che governano l'orientamento. Per garantire risultati più robusti nella fase di allineamento è stato disegnato un pattern adesivo applicato sul calibratore, geometricamente e cromaticamente non ripetitivo, dotato di target codificati.

II sistema ha previsto, infine, l'alloggiamento del 3Dino Plate su un binario a scorrimento, che permettesse la traslazione, manuale in questa prima sperimentazione, della piastra di calibrazione rispetto al microscopio (fig. 2).

I microscopi digitali Dino-Lite forniscono una soluzione potente, portatile e funzionale per l'ispezione di dettaglio. I modelli oggetto di sperimentazione, l'AM4 I I 3ZT Universal [3] e l'AM739 I 5MZTL High Speed, appartengono a due classi diverse, sia in termini di caratteristiche tecniche che di special features (fig. 3), e, di conseguenza, con fasce di prezzo abbastanza differenti. L'elemento che distingue nettamente i due microscopi è la Working Distance - WD, owero la distanza lineare tra l'estremità dell'ugello del microscopio e l'oggetto da rilevare (fig. 4). La Working Distance incide direttamente sull'ingrandimento (Magnification Factor - MF), sull'area inquadrabile (FOV) e sulla profondità di campo (DOF) dell'immagine acquisita. E banale osservare che in fotogrammetria è importante stabilire un giusto compromesso fra questi fattori, tenendo a mente che più si è lontani dal soggetto (WD elevata), più basso 
Fig. 3. Specifiche tecniche dei modelli di microscopio della DinoLite impiegati.
Fig. 4. 'Working Distance' del microscopio High-Speed' (a sinistra, pari a 75,5 mm) e del microscopio 'AM4 I I 3ZT Universal' (a destra, pari a $21,7 \mathrm{~mm}$ ), alloggiati sul sostegno 'Dino-Lite RK-I0-EX' e operanti con un medesimo

ingrandimento (30x) In dettaglio l'acquisizion. di una, lativa porizione din libeliva porzione particolare del un particolare del (ZOOM)

\begin{tabular}{|c|c|c|}
\hline Model & AM4113ZT Universal & AM73915MZTL High Speed \\
\hline Dino-Lite range & Dino-Lite Universal & Dino-Lite High Speed \\
\hline Diffuser available & MSAA111A2 (optional accessory) & Yes \\
\hline Polarizer & Yes, linear & Yes, linear \\
\hline Magnification & $10-70 x, 200 x$ & $10-140 x$ \\
\hline Working Distance & Standard & Long \\
\hline Resolution & 1.3 Megapixel (1280x1024) & 5 Megapixel (2592x1944) \\
\hline Maximum Frame rate & 30 fps & 45fps (max 20fps for video recording) \\
\hline Interface & USB 2.0 & USB 3.0 \\
\hline Housing material & Composite/plastic housing & Metal housing \\
\hline & No & Aut. Magn. Reading \\
\hline Special features & & $\begin{array}{c}\text { Extended Dynamic Range (EDR) } \\
\text { Flexible LED Control (FLC) }\end{array}$ \\
\hline Price range & $€ 200,00-€ 350,00$ & \begin{tabular}{c} 
E1000,00 $-€ 1250,00$ \\
\hline
\end{tabular}
\end{tabular}

sarà l'ingrandimento, con un più ampio campo visivo e più profondità di campo; al contrario, più si è vicini al soggetto (WD esigua), maggiore sarà l'ingrandimento, con un campo visivo più piccolo e una profondità minore.

La Working Distance è pertanto un parametro di estrema importanza dal punto di vista pratico e lavorando con diversi microscopi (fig. 5), costringe, inevitabilmente, all'impiego di diversi ingrandimenti [4] in fase operativa.

Sia il modello Universal che il modello High Speed sono stati combinati con il calibratore, fissando il microscopio nell'apposito alloggiamento del sostegno Dino-Lite RK-I O-EX e collegandolo a una workstation portatile. Le acquisizioni, quindi, sono state poi condotte con gli ingrandimenti riassunti in fig. 5, operando con la procedura dedicata di DinoCapture 2.0 (<www.dino-lite.eu/index.php/it/software-dino-lite>) e valutando, per l'occasione, anche gli algoritmi Extended Dynamic Range - EDR ed Extended Depth Of Field - EDOF, che appro-

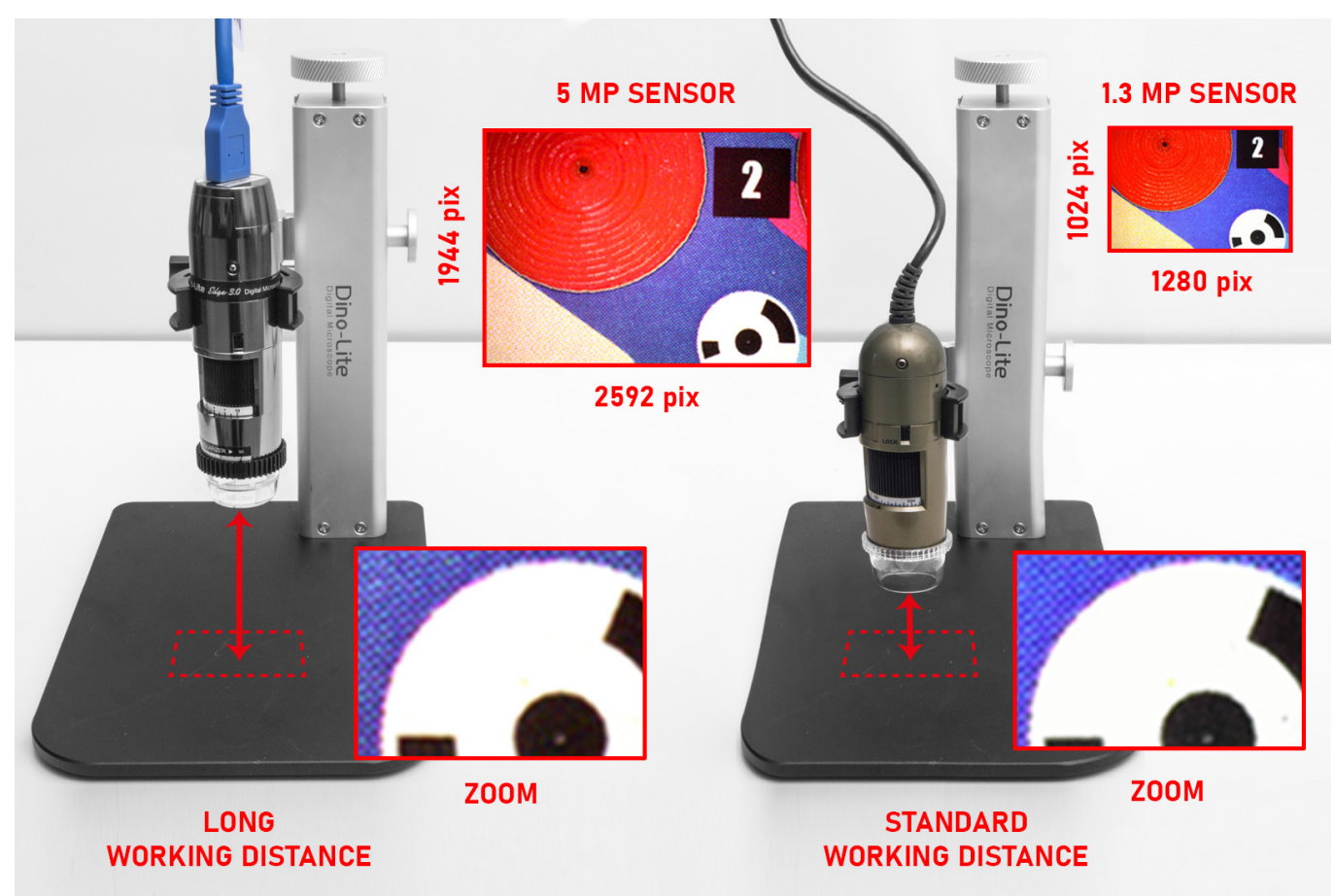




\begin{tabular}{|c|c|c|c|c|c|c|}
\hline & \multicolumn{3}{|c|}{ AM4113ZT Universal } & \multicolumn{3}{c|}{ AM73915MZTL High Speed } \\
\hline & $\mathbf{2 0 x}$ & $\mathbf{4 0 x}$ & $\mathbf{5 0 x}$ & $\mathbf{2 0 x}$ & $\mathbf{4 0 x}$ & $\mathbf{9 0 x}$ \\
\hline $\begin{array}{c}\text { Working } \\
\text { Distance }\end{array}$ & $48,7 \mathrm{~mm}$ & $9,0 \mathrm{~mm}$ & $1,9 \mathrm{~mm}$ & $115,0 \mathrm{~mm}$ & $56,8 \mathrm{~mm}$ & $32,7 \mathrm{~mm}$ \\
\hline $\begin{array}{c}\text { Field Of } \\
\text { View }\end{array}$ & $19,8 \times 14,9 \mathrm{~mm}$ & $9,9 \times 7,4 \mathrm{~mm}$ & $7,9 \times 5,9 \mathrm{~mm}$ & $39,0 \times 29,2 \mathrm{~mm}$ & $9,8 \times 7,3 \mathrm{~mm}$ & $4,3 \times 3,2 \mathrm{~mm}$ \\
\hline $\begin{array}{c}\text { Depth Of } \\
\text { Field }\end{array}$ & $3,6 \mathrm{~mm}$ & $1,4^{* *} \mathrm{~mm}$ & $0,88 \mathrm{~mm}$ & $12,0 \mathrm{~mm}$ & $1,8 \mathrm{~mm}$ & $0,37 \mathrm{~mm}$ \\
\hline
\end{tabular}

fondiremo nel prossimo paragrafo. I set di immagini sono stati così elaborati in un software di tipo SfM, Agisoft Metashape, secondo il generale workflow fotogrammetrico; le prime acquisizioni non hanno tenuto in conto della variazione di distanza focale correlata ai diversi gradi di magnificazione (MF), sfruttando le nuove possibilità offerte dai software SfM di ricostruire geometrie accurate anche in funzione di dataset meno rigorosi.

\section{Analisi dei dati}

Le valutazioni dei dati restituiti con la strumentazione microscopica hanno, in primo luogo, tenuto in considerazione le procedure di gestione software delle prese micro-fotogrammetriche (fig. 6) in ambiente Agisoft Metashape.

I microscopi digitali portatili, allo stato, a differenza delle camere fotografiche, rivelano alcune problematiche specifiche per le applicazioni fotogrammetriche: tra le più evidenti, e più limitanti, l'assenza di dati Exif. Queste informazioni associate alla matrice di pixel, come ben noto, conservano dati relativi al modello di camera impiegato, alle dimensioni del sensore e alla lunghezza focale e sono variabili essenziali, in assenza di una calibrazione di laboratorio, utilizzate da qualsiasi software per sviluppare i calcoli adeguati alla stima della distorsione e al posizionamento delle prese. Oltre l'assenza di tali informazioni va aggiunta la limitata profondità di campo di questi strumenti: la possibilità offerta dalla modalità Extended Depth Of Field - EDOF di superare questo limite acquisendo e combinando più scatti su differenti piani di messa a fuoco, è risultata non migliorativa in termini di qualità delle immagini, ovvero poco adatta e risolutiva al caso di studio (con questa modalità non è possibile, ad esempio, stimare un valore univoco della distanza focale per la singola presa). L'altra modalità sperimentata, Extended Dynamic Range - EDR, pur non offrendo una soluzione alla ridotta profondità di campo, sovrapponendo immagini con differenti esposizioni, si è rivelata utile per caratterizzare i dettagli di aree più scure o più luminose, evidenziando una particolare efficacia per superfici riflettenti, nonostante i microscopi utilizzati già beneficiassero della dotazione di una luce polarizzata. Va tenuto in conto che le due modalità, Extended Depth Of Field - EDOF ed Extended Dynamic Range - EDR, richiedono un tempo di acquisizione superiore (di circa 20 secondi per ogni singolo scatto rispetto alla modalità Normal), per la preelaborazione del dato effettuata direttamente nell'applicativo proprietario DinoCapture 2.0 (fig. 7).

Già a valle di un'analisi speditiva è stato possibile valutare come apprezzabili i risultati ottenuti dal modello Universal (fig. 8), che ha garantito output comparabili al modello High Speed, nonostante

\begin{tabular}{|c|c|c|c|c|c|c|c|}
\hline Model & Mode & $\begin{array}{c}\text { Aligned } \\
\text { Cameras }\end{array}$ & $\begin{array}{c}\text { Ground } \\
\text { resolution } \\
\text { (GSD) }\end{array}$ & $\begin{array}{c}\text { Tie } \\
\text { Points** }\end{array}$ & Projections & $\begin{array}{c}\text { RMS } \\
\text { reprojection } \\
\text { error }\end{array}$ & $\begin{array}{c}\text { Control } \\
\text { Points } \\
\text { Error }\end{array}$ \\
\hline $\begin{array}{c}\text { AM4113ZT } \\
\text { Universal }\end{array}$ & Normal & $110 / 111$ & $4,01 \mu \mathrm{m} /$ pix & 145.613 & 411.610 & 1,47 pix & $0,076 \mathrm{~mm}$ \\
\hline & EDOF & $134 / 135$ & $6,49 \mu \mathrm{m} / \mathrm{pix}$ & 104.520 & 266.653 & 1,01 pix & $0,094 \mathrm{~mm}$ \\
\hline $\begin{array}{c}\text { AM73915MZTL } \\
\text { High Speed }\end{array}$ & EDR & $100 / 103$ & $3,45 \mu \mathrm{m} /$ pix & 171.702 & 471.944 & 0,77 pix & $0,094 \mathrm{~mm}$ \\
\cline { 2 - 9 } & Normal & $148 / 152$ & $4,44 \mu \mathrm{m} / \mathrm{pix}$ & 169.503 & 455.609 & 1,77 pix & $0,089 \mathrm{~mm}$ \\
\hline
\end{tabular}


Fig. 7. 'DinoCapture 2.0 acquisition' e 'Agisoft Metashape' processing time comparison:le elaborazioni sono stata portate a termine con la medesima workstation grafica dotata di CPU Intel $199900 k$, GPU RTX 2080 Ti e 64GB di RAM.

\begin{tabular}{|c|c|c|c|c|c|c|c|c|c|}
\hline Model & $\begin{array}{l}\text { Operative } \\
\text { Mode }\end{array}$ & Acquisition & Alignment & $\begin{array}{c}\text { Marker } \\
\text { positioning }\end{array}$ & $\begin{array}{c}\text { Dense } \\
\text { Point } \\
\text { Cloud }\end{array}$ & Model & DSM & Ortho & TOT \\
\hline $\begin{array}{l}\text { AM4113ZT } \\
\text { Universal }\end{array}$ & Normal & $15^{\prime}$ & $1^{\circ} 08^{\prime}$ & $30^{\prime}$ & $17^{\prime}$ & $30^{\prime}$ & 8" & $46^{\prime \prime}$ & $2^{\circ} 41^{\prime}$ \\
\hline \multirow{3}{*}{$\begin{array}{l}\text { AM73915MZTL } \\
\text { High Speed }\end{array}$} & $\begin{array}{c}\text { Extended } \\
\text { Depth Of } \\
\text { Field } \\
\text { EDOF }\end{array}$ & $45^{\prime}$ & $1^{\circ} 56^{\prime}$ & $30^{\prime}$ & 9' & $40^{\prime}$ & $11 "$ & $1^{\prime}$ & $4^{\circ} 02^{\prime}$ \\
\hline & $\begin{array}{c}\text { Extended } \\
\text { Dynamic } \\
\text { Range } \\
\text { EDR }\end{array}$ & $45^{\prime}$ & $58^{\prime}$ & $30^{\prime}$ & $11^{\prime}$ & $18^{\prime}$ & 11" & $35 "$ & $2^{\circ} 43^{\prime}$ \\
\hline & Normal & $15^{\prime}$ & $2^{\circ} 13^{\prime}$ & $30^{\prime}$ & $11^{\prime}$ & $1^{\circ} 02^{\prime}$ & $11 "$ & 1 ' & $4^{\circ} 12^{\prime}$ \\
\hline
\end{tabular}

sia da considerare entry-level rispetto a quest'ultimo. Dalle analisi e dai grafici di cui alle precedenti tabelle e immagini, particolarmente esemplificativo è lo studio di una slice di nuvola di punti esportata dai quattro modelli 3D omologhi: pur non potendo stimare in maniera assoluta le incertezze (alla sottomissione del lavoro ancora non è stato portato a termine un rilievo di tipo range-based che potrà essere assunto come modello di riferimento) è comunque evidente il dettaglio della nuvola restituita dal modello Universal (fig. 9, I0). Tuttavia, nonostante le conferme circa le performance della strumentazione testata, i dati in possesso non permettono, per ora, valutazioni più spinte e analitiche sull'accuratezza del sistema, il che renderà necessario svolgere nuovi studi per risalire, finalmente, alle variabili parametrali dell'orientamento interno.

Ulteriori approfondimenti saranno condotti, quindi, confrontando i risultati delle procedure SfM con altri sistemi di rilevamento per l'assunzione di una superficie di riferimento da impiegare come modello 'assoluto' di comparazione.

\section{Considerazioni e sviluppi futuri}

Le esperienze compiute sono confacenti a definire come valido l'impiego di microscopi portatili a fini fotogrammetrici. L'effettiva risoluzione del dato, unito al rapporto qualità/prezzo di queste strumentazioni a cui si aggiungono le caratteristiche di portabilità e la relativa facilità d'uso, rappresenta senza dubbio il punto di forza del metodo. D'altro canto, è doveroso

\begin{tabular}{|c|c|c|c|c|c|c|c|c|}
\hline \multirow{2}{*}{ Model } & \multirow{2}{*}{ Mode } & \multicolumn{2}{|c|}{ Dense Point Cloud } & \multicolumn{2}{|c|}{ Model } & \multirow{2}{*}{$\begin{array}{c}\text { DEM } \\
\begin{array}{c}\text { Elevation } \\
\text { Range }\end{array}\end{array}$} & \multicolumn{2}{|c|}{ Ortho } \\
\hline & & Points & Faces & Vertices & $\begin{array}{c}\text { Point } \\
\text { Density }\end{array}$ & & Size & $\begin{array}{l}\text { Image } \\
\text { Weight }\end{array}$ \\
\hline 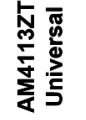 & Normal & 32.148 .558 & 6.429 .711 & 3.222 .831 & $\begin{array}{c}6,23 \times 10^{4} \\
\text { points } / \mathrm{mm}^{2}\end{array}$ & $3,0 \mathrm{~mm}$ & $\begin{array}{c}3850 \times 3893 \\
\text { pix }\end{array}$ & $\begin{array}{l}60,1 \\
M B\end{array}$ \\
\hline \multirow{3}{*}{ 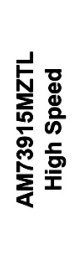 } & EDOF & 49.018 .983 & 9.803 .616 & 4.949 .755 & $\begin{array}{c}6,49 \times 10^{4} \\
\text { points } / \mathrm{mm}^{2}\end{array}$ & $7,5 \mathrm{~mm}$ & $\begin{array}{c}6026 \times 5848 \\
\text { pix }\end{array}$ & $\begin{array}{l}85,2 \\
M B\end{array}$ \\
\hline & EDR & 24.577 .548 & 4.915 .506 & 2.465 .745 & $\begin{array}{c}8,41 \times 10^{4} \\
\text { points } / \mathrm{mm}^{2}\end{array}$ & $2,8 \mathrm{~mm}$ & $\begin{array}{c}6043 \times 5879 \\
\text { pix }\end{array}$ & 77,8 \\
\hline & Normal & 48.697 .228 & 9.739 .215 & 4.911 .372 & $\begin{array}{c}5,08 \times 10^{4} \\
\text { points } / \mathrm{mm}^{2}\end{array}$ & $6,0 \mathrm{~mm}$ & $\begin{array}{c}6265 \times 6108 \\
\text { pix }\end{array}$ & $\begin{array}{c}106,0 \\
M B\end{array}$ \\
\hline
\end{tabular}


Fig. 9. Modelli generati con diversi dataset: il primo acquisito con microscopio 'AM4I I 3ZT Universal' in modalità Normal (ovvero in assenza "special features"); il secondo, terzo e quarto modello sono relativi invece, alle acquisizion con il microscopio "AM739I5MZTL High "AM739I5MZ LL High Speed "rispettivamente Normal. II 'White Balance' Normal. II 'White Balan
è stato impostato per è stato impostato per
un'analisi automatica del colore, senza correzioni posteriori delle immagini, determinando, pero, un'evidente differenza nella restituzione del dato RGB con il modello 'AM4I I 3ZT Universal' (tendente al verde in alto a sinistra), rispetto a quelli del modello 'AM739I5MZTL High Speed' (più corretti cromaticamente).
Fig. 10. A sinistra, confronto di sezioni di nuvole di punti generate con diversi dataset: la prima da microscopio 'AM4I I 3ZT Universal' in modalità Normal (ovvero in assenza di 'special features') la seconda, terza e quarta sono relative, invece, alle nuvole di punti da microscopio AM739I5MZTL High Speed', rispettivamente in modalità EDOF, EDR e Normal. A destra la nuvola di punti ottenuta con il microscopio con il microscopio posizionamento della slice scelta per il suddetto confronto.
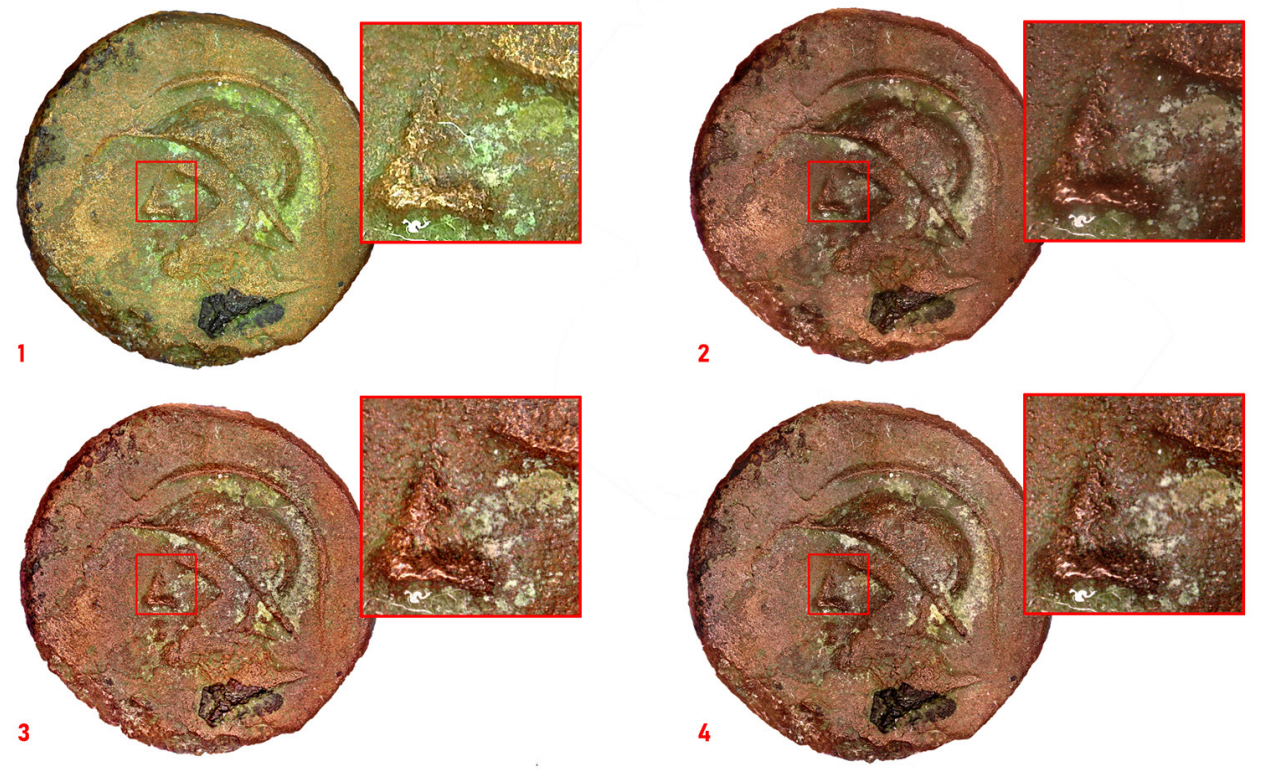

specificare che, al fine di perseguire l'obiettivo primario di ogni rilievo, cioè la verificabilità e riproducibilità del valore metrico, sono ancora da meglio definire alcuni aspetti nodali.

Oltre a modifiche di natura software, che permettano il salvataggio dei dati Exif, si sono rese evidenti alcune modifiche necessarie al calibratore di figura I. In primo luogo, nello spessore del 3Dino Plate, che dovrà essere minore rispetto a quello proposto: questo permetterà la riduzione dell'interasse dei marker, consentendo l'individuazione di più punti di coordinate note e quindi di avere, oltre ai Control Points, anche dei Check Points. Si è osservata, inoltre, l'opportunità di modificare la geometria della piastra, da rettangolare a rotonda, per poterla adattare a un piatto rotante motorizzato, così da facilitare possibili acquisizioni ad asse inclinato, oltre a dotarla di sporgenze su più livelli offrendo più piani di messa a fuoco e un mezzo più rigoroso per la valutazione delle profondità. In aggiunta, la procedura di presa andrà riconsiderata in termini di ingrandimento, ovvero magnificazione, bloccando la ghiera
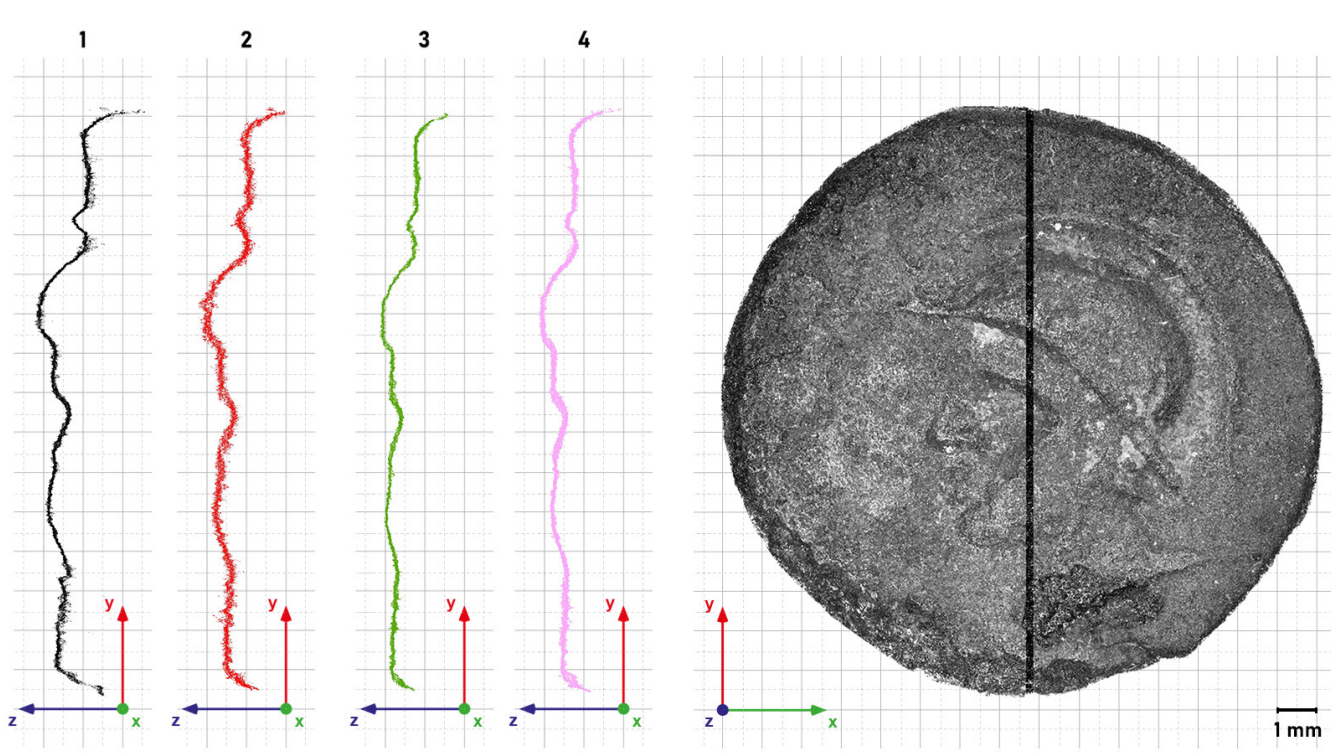
Fig. I I. Scostamenti globali e relativi rispetto ai singoli assi, espressi in millimetri, dei modelli 3D restituiti. I dati provenient dal microscopio 'Universal' sono stati assunti come riferimento per cosi graficizzare gli per cosi graficizzare gi scarti delle restituzion del microscopio 'High Speed' (in modalita Normal, EDOF o EDR).
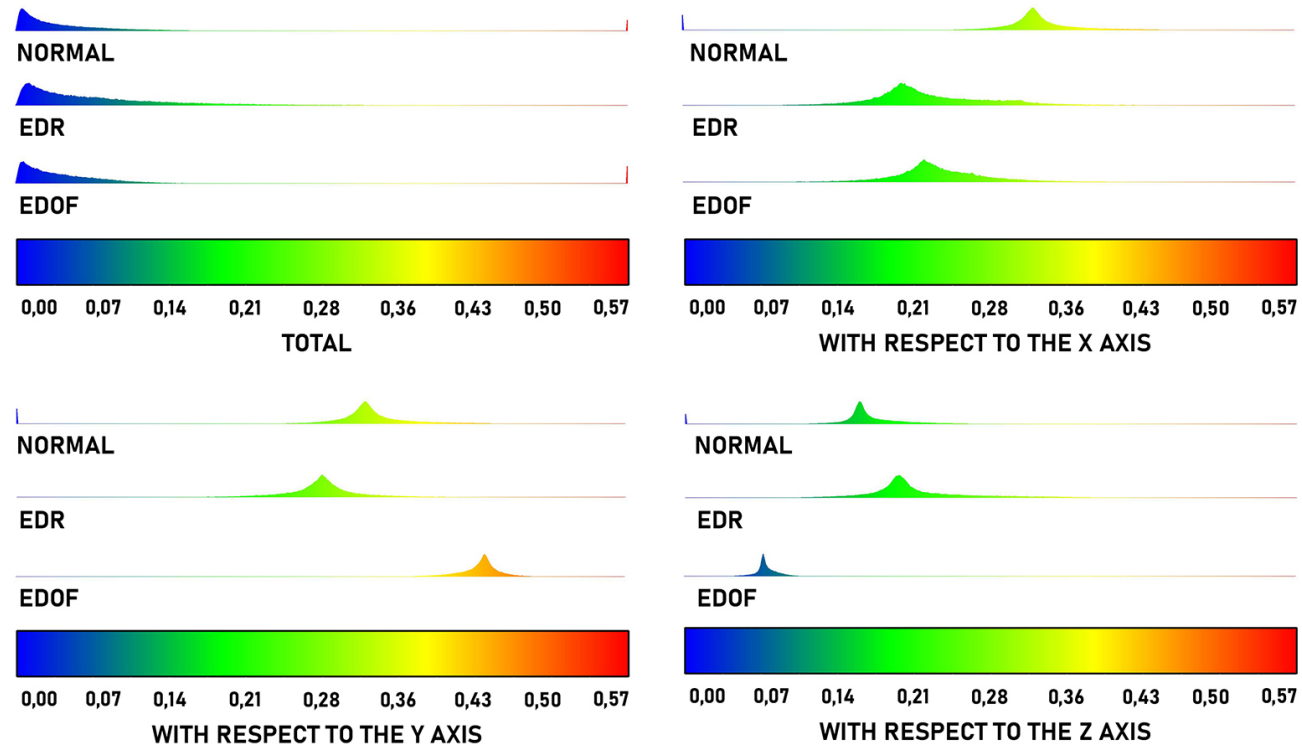

del microscopio che consente di modificare il Magnification Factor - mantenendo, quindi, unica e fissa la distanza focale - e avvicinandosi e/o allontanandosi dall'oggetto, ovvero modificando la Working Distance associata, sempre garantendo una sufficiente sovrapposizione (cioè operando diversamente dal combinare prese con differenti MF eWD).

Nonostante le problematiche, nel quadro delle moderne micro-tecnologie, prevalgono da questo work in progress più spunti interessanti per la documentazione tridimensionale di piccolissimi manufatti e tessere altrimenti difficilmente rappresentabili.

Fig. 12. Dense Surface Model generati con i diversi dataset: il primo acquisito con microscopio 'AM4I I 3ZT Universa' in modalità Normal (ovvero in assenza di 'special features'); il secondo, terzo e quarto modello sono relativi, invece, alle acquisizioni con il microscopio AM739I5MZTi High 'AMP', rispettiva High Speed, rispettivamente in modalita EDOF, EDR Normal.
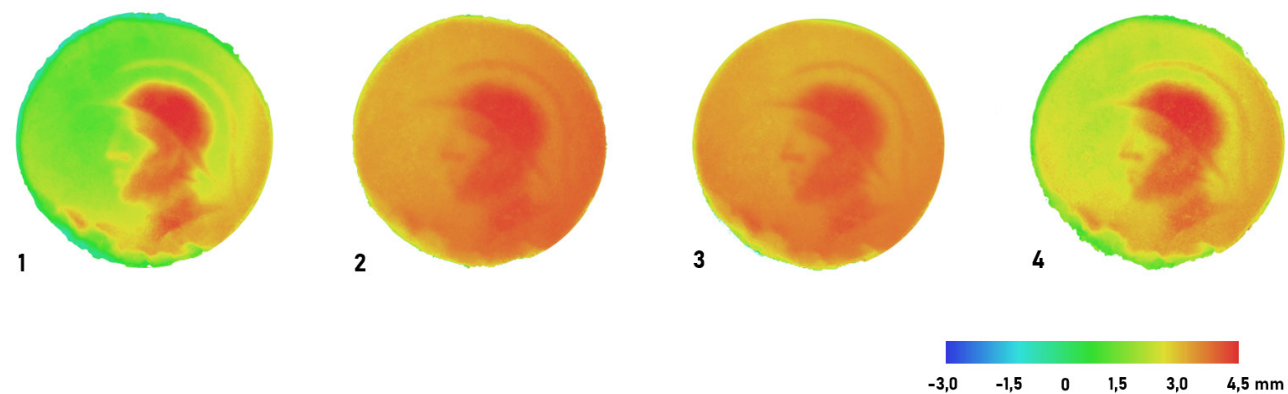

\section{Ringraziamenti}

Ringraziamo, sinceramente, la IDCP Digital Innovation - nelle persone di Jan Boers, Danielle van Duijvendijk e Ivo Manders - e ovviamente, la Dino-Lite Digital Microscope per il supporto, la disponibilità e le attrezzature messe a disposizione per la ricerca. Ringraziamo, infine, Giacomo Pardini del Dipartimento di Scienze del Patrimonio Culturale dell'Università di Salerno per aver permesso l'accesso all'archivio numismatico e per aver supportato la pubblicazione di questi risultati.

\section{Note}

[I] L'emissione di Velia Atena/Tripode è in studio da parte di Federico Carbone, nell'ambito del progetto di ricerca sui rinvenimenti monetali da Velia dal titolo La moneta di basso conto a Elea/Velia: uso e produzione (diretto da Renata Cantilena) e del progetto Art Bonus Le monete di Elea/Velia - Un restauro per la conoscenza e la valorizzazione del patrimonio archeologico della città di Parmenide, svolto in collaborazione con la Soprintendenza Archeologia, Belle Arti e Paesaggio per le province di Salerno e Avellino e sostenuto dalla Fondazione Nazionale delle Comunicazioni (Roma).

[2] Sono di seguito riportati i settaggi della stampante 3D Creality CR- IO v 1.0 per la realizzazione del 3Dino Plate: temperatura di letto $50^{\circ} \mathrm{C}$; temperatura dell'ugello $200^{\circ} \mathrm{C}$; diametro dell'ugello $0,3 \mathrm{~mm}$; materiale utilizzato PLA I,75 mm +/- $0,05 \mathrm{~mm}$; altezza di layer $0,12 \mathrm{~mm}$ infill $20 \%$; infill pattern cubic; velocità di stampa $50 \mathrm{~mm} / \mathrm{s}$. II tempo occorso è stato di circa 13 ore. Le caratteristiche intrinseche del PLA rendono le stampe realizzate con questo materiale soggette a un 
deterioramento relativamente rapido dovuto all'assorbimento dell'umidità e alla sensibilità ai raggi UV; ciò conduce gli autori a considerare valida la scelta di questo materiale solo per la fase di prototipazione della piastra. Con la stampante EOSINT M270 Xtended version è in realizzazione un prototipo in acciaio del 3Dino Plate.

[3] II modello AM4 I I 3ZT Universal è stato oggetto della precedente pubblicazione, di cui questo contributo rappresenta la naturale prosecuzione [Antinozzi, Ronchi, Barba 2020].

[4] La volontà di ottenere un dettaglio maggiore spinge all'utilizzo di ingrandimenti elevati, ma la differente Working Distance dei due microscopi non sempre ha permesso l'impiego dei medesimi valori di ingrandimento. Ad esempio, valori di ingrandimento vicini al 50x avrebbero reso quasi necessario il contatto del microscopio AM4 I / $3 Z$ T Universal con la superficie della moneta.

\title{
Riferimenti bibliografici
}

Adamopoulos E., Rinaudo F. (2019). An Updated Comparison on Contemporary Approaches for Digitization of Heritage Objects. In Catelani M., Daponte P. (a cura di). Atti di 2019 IMEKO TC-4 International Conference on Metrology for Archaeology and Cultural Heritage. Firenze, Italia, 4-6 Dicembre, 2019, pp. I-6.

Antinozzi S., Ronchi D., Barba S. (2020). Macro e micro fotogrammetria per la virtualizzazione della laminetta orfica (V-IV a.C.) del Museo Nazionale di Vibo Valentia / Macro and Micro Photogrammetry for the Virtualization of the Orphic Foil (V-IV B.C) of National Museum of Vibo Valentia. In Arena A. et al. (a cura di). Connettere. Un disegno per annodare e tessere. Atti del $42^{\circ} \mathrm{Convegno}$ Internazionale dei Docenti delle Discipline della Rappresentazione / Connecting. Drawing for weaving relationships. Proceedings of the 42 nd International Conference of Representation Disciplines Teachers. Milano: Franco Angeli, pp. I 538 - I 555.

Apollonio F.I., Fantini F., Garagnani S., Gaiani M.A. (202I). Photogrammetry-Based Workflow for the Accurate 3D Construction and Visualization of Museums Assets. In Remote Sensing, vol. I3, Issue 3, 486, pp. I-40.

Bolognesi C. M., Fiorillo F. (20 I8). Optimization of texture mapping process in the Reality-Based Modeling application. In Salerno R. (a cura di). Rappresentazione / Materiale / Immateriale. Atti del $40^{\circ}$ Convegno internazionale dei Docenti delle Discipline della Rappresentazione. Milano, Italia, I5-I7 settembre, 20 I8, pp. 337-342.

Esmaeili F., Ebadi H. (20 I7). Handy Microscopic Close-RangeVideogrammetry. In The International Archives of the Photogrammetry, Remote Sensing and Spatial Information Sciences, vol. XLII-4/W4, pp. 65-67.

Galantucci L.M., Pesce M., Lavecchia F. (2016). A powerful scanning methodology for 3D measurements of small parts with complex surfaces and sub millimeter-sized features, based on close range photogrammetry. In Precision Engineering, vol. 43, pp. $211-219$.

Gontard L. C. Schierholz R.,Yu S., Cintas J., Dunin-Borkowski R. E. (2016). Photogrammetry of the three-dimensional shape and texture of a nanoscale particle using scanning electron microscopy and free software. In Ultramicroscopy, vol. 169, pp. 80-88.

Hansen H.N., Carneiro K., Haitjema H., De Chiffre L. (2006). Dimensional Micro and Nano Metrology. In CIRP Annals, vol. 55, Issue 2, pp. 72 I-743.

Hess M., MacDonald L.W., Valach J. (20 8). Application of multi-modal 2D and 3D imaging and analytical techniques to document and examine coins on the example of two Roman silver denarii. In Heritage Science, vol. 6, Issue 5, pp. I-22.

Mitchell H. L., Kniest H.T. (1999). Digital Photogrammetry and Microscope Photographs. In Photogrammetric Record, n. I 6, pp. 695-704.

Morena S., Barba S., Álvaro-Tordesillas A. (2019). Shining 3D Einscan-Pro, application and validation in the field of Cultural Heritage, from the Chillida-Leku Museum to the Archaeological Museum of Sarno. In The International Archives of the Photogrammetry, Remote Sensing and Spatial Information Sciences, vol. XLII-2/W I8, pp. I 35- I 42.

Pieraccini M., Guidi G., Atzeni C. (200 I ). 3D digitizing of cultural heritage. In Journal of Cultural Heritage, n. 2, pp. 63-70.

Plisson H., Zotkina L.V. (20I5). From 2D to 3D at macro- and microscopic scale in rock art studies. In Digital Applications in Archaeology and Cultural Heritage, vol. 2, Issues 2-3, pp. 102-1 19.

Tolksdorf J. F., Elburg R., Reuter T. (2017). Can 3D scanning of countermarks on Roman coins help to reconstruct the movement of Varus and his legions. In Journal of Archaeological Science: Reports, vol. I I, pp. 400-4I 0.

\author{
Autori \\ Sara Antinozzi, Università degli Studi di Salerno, santinozzi@unisa.it \\ Diego Ronchi, Università degli Studi di Salerno, dronchi@unisa.it
}

Salvatore Barba, Università degli Studi di Salerno, sbarba@unisa.it

Per citare questo capitolo: Antinozzi Sara, Ronchi Diego, Barba Salvatore (202I). 3Dino System, come accorciare le distanze nei rilievi di precisione/3Dino System, Shortening Distances in Precision Surveys. In Arena A. Arena M. Mediati D. Raffa P. (a cura di). Connettere. Un disegno per annodare e tessere Linguagri Distanze Tecnologie. Atti del $42^{\circ}$ Convegno Internazionale dei Docenti delle Discipline della Rappresentazione/Connecting Drawing for weaving relationship. Languages Distances Technologies. Proceedings of the 42th International Conference of Representation Disciplines Teachers. Milano: FrancoAngeli, pp. $1922-1941$. 


\title{
3Dino System, Shortening Distances in Precision Surveys
}

\author{
Sara Antinozzi \\ Diego Ronchi \\ Salvatore Barba
}

Abstract

Rapid succession of technological innovations for cultural heritage digitization, as well as the uncertainty on the conditions and continuity of its fruition - with evident reference to asset's conservation, but also to the recent inaccessibility conditions-, are imposing a deepening for structured procedures of three-dimensional survey and virtualization.

These procedures, mainly addressed to orders of magnitude and scales of restitution for architecture, are however not oriented towards the codification and formalization of relevant practices for very small objects; this issue is made even more compelling by the need of acquiring highly uniform and/ or reflective geometries and surfaces which, as known, make the recognition of homologous points more complex in Structure from Motion algorithms.

The tests we conducted with Dino-Lite portable digital microscopes verified the potential of using 'micro' images also for Cultural Heritage documentation, highlighting, however, the need for some technological developments, especially related to depth of field and consequent acquisition geometry. This paper aims at solving some problems increasing the performance of microscopic photogrammetry by optimizing the acquisition procedures with the design of customized accessories for micro-photogrammetry. The results were pursued as part of a technology transfer agreement aimed at advancing a protocol for photogrammetric documentation of small artefacts.

Keywords

Dino-Lite handheld microscope, cultural heritage, small artefacts, ancient coinage, micro-photogrammetry.

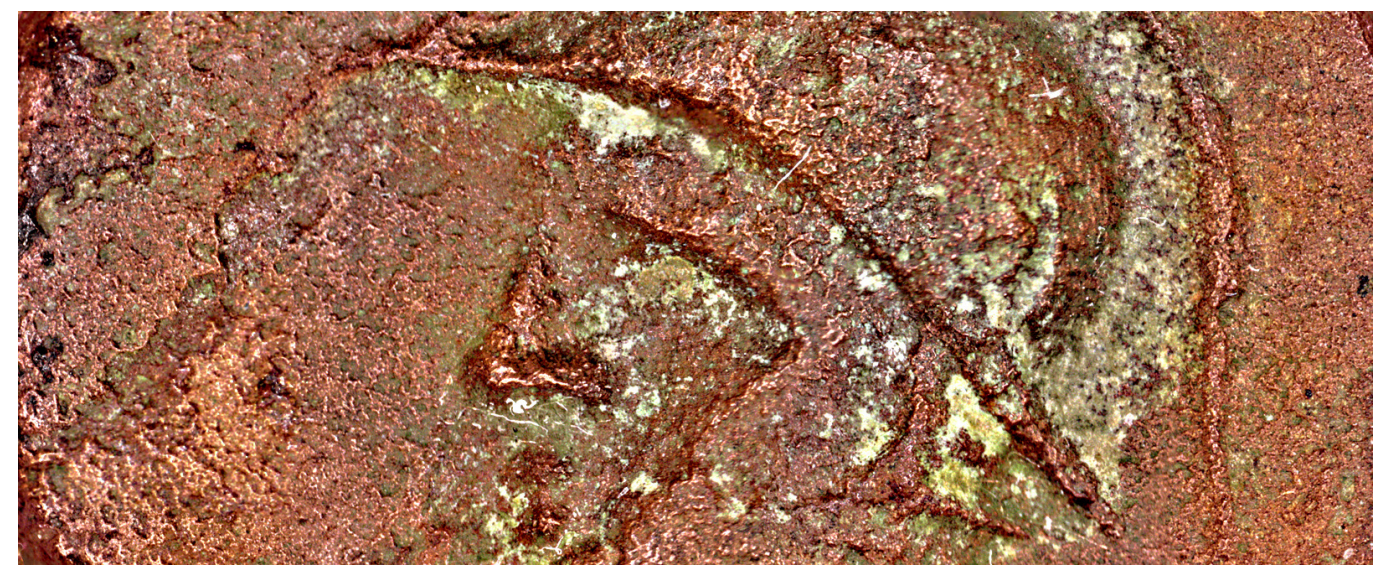




\section{Introduction}

Sensors, acquisition techniques and computational systems continuous improvement constitutes the recording and visualizing data tools real strength [Adamopoulos, Rinaudo 2019, pp. 1-6]. This assumption facilitated the introduction of increasingly automated processes, with increasing accuracy, characterized by limited times and costs; circumstance that thickens the network of complementarity and interaction relationships among various disciplines related to imaging and three-dimensional processing, including manufacturing industry, medical sciences, entertainment and, obviously, cultural heritage [Pieraccini, Guidi, Atzeni 200I, pp. 63-70].

The increased need for three-dimensional measurements of objects with complex surfaces and sub-millimetre morphological characteristics generated various range-based solutions for micrometric applications [Hansen et al. 2006, pp. 721-743]. However, not infrequently, both optical and mechanical limitations and non-negligible costs directed the interest towards precision photogrammetry, a rapid, accurate and cheaper paradigm, suitable for surveying both small and complex surfaces [Galantucci, Pesce, Lavecchia 2016, pp. 2II-219], although often to the detriment of the reconstruction of the entire sample's volume [Gontard et al. 20 I6, pp. 80-88]. The advent of portable digital microscopes developed for inspection, documentation, and metrological digital analysis -already popular in the production and quality control industry, as well as in the medical field- revealed a not negligible potential for modelling small objects, reaching accuracies of the order of a tenth of a millimetre [Esmaeili, Ebadi 2017, pp. 65-67]. Within a few years, the possibility of using photographs taken by a microscope attracted the attention of sectors distant from the production-industrial one, such as that of cultural heritage. Among the most compelling reasons for this interest is the need to make accessible many finds, often very small, lying in the warehouses of our museums, also due to the limited exhibition spaces. This need has recently been added to the unavailability of the visit in person at the exhibition site. In addition to the urgency of exhibiting and cataloguing these objects, the use of digital 3D representations would introduce significant improvements in the work of Cultural Heritage professionals, framing the asset in a global and holistic perspective, inserting it into a vast system of conservation of information, which can be modified and implemented at the same time [Apollonio et al. 2021, pp. I-40].

Addressing small finds survey determines not only a change in the scale of representation, but also an acquisition operating system rethinking, also operating in situations

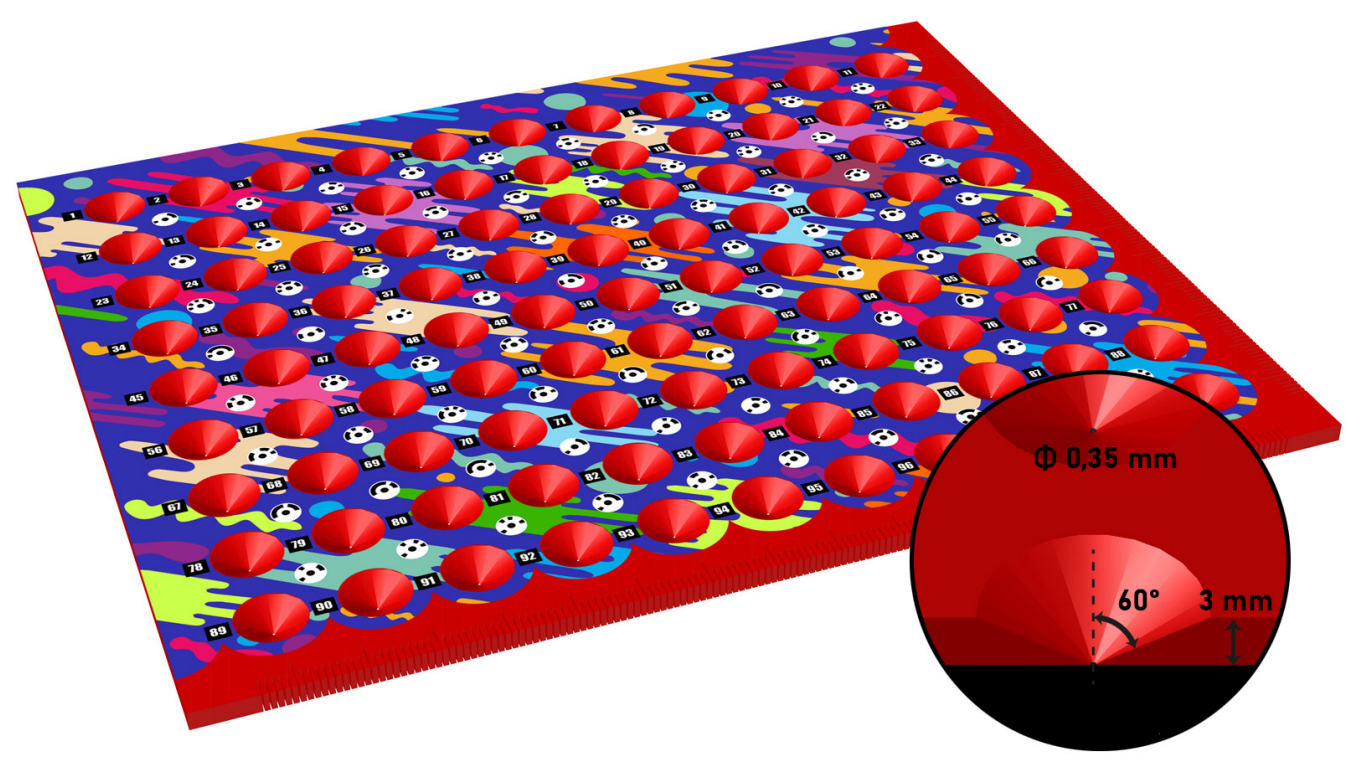


that are often not easy [Plisson, Zotkina 20 I5, pp. I02-I I9]. The use of microscopes for photogrammetric purposes, difficult due to the narrow field of view and reduced depth of field characterizing the optical system, makes -in fact- an innovative challenge for adapting these instruments to the peculiarities of our scientific disciplinary sector. Thus, as in other areas of close-range photogrammetry, the advantage of digital microscopic photogrammetric processing will lie, once obtained the full control of all system variables, on the one hand in encoding an automation of the acquisition process. [Mitchell, Kniest 1999, pp. 695-704] and on the other hand in pursuing adequate and verifiable accuracy.

Currently on the market some range-based instruments are already available meeting the illustrated needs, but at not always low costs [Tolksdorf, Elburg, Reuter 20 I7, pp. 400-4I 0] and not so effective in re-positioning single pixels on the surface when compared to image-based systems [Bolognesi, Fiorillo, 20 I 8, pp. 337-342], or integrated approaches still representing the most effective solution [Morena, Barba, Álvaro-Tordesillas, 20 I9, pp. I35- I 42]. The contribution, therefore, will focus on the systematization of hardware tools, individually already available, to explore microscopic photogrammetry to define a standardized procedure for the acquisition of very small objects with complex details and textures.

\section{Case study: surveying a I ${ }^{\text {st }}$ Century BC bronze coin}

The precision survey of a bronze coin from Velia, an ancient polis of Magna Graecia today belonging to the Archaeological Park of Paestum and Velia, represented a somewhat emblematic opportunity to experience the photogrammetric performance of some digital microscopes.

The needs expressed by ancient numismatics studies, especially for coinage examination, lead not to rely solely on traditional documentation and analysis, consisting mainly of manual measurements and photographs; a type of documentation that can be limiting in terms of information acquisition and subsequent analytical approaches. More advanced methods for recording and examining the find are gradually establishing themselves in conservation practices and in the museum environment, becoming almost the norm today [Hess, MacDonald, Valach 2018, pp. I-22].

The object, selected to determine whether the proposed digital recording methods can support the research results, is a coin approximately $1.2 \mathrm{~cm}$ in diameter, weighing $2.93 \mathrm{~g}$ (inventory number 223964, suitable for size and surface's nature), which on the obverse has a helmeted Athena's head turned to the left and on the reverse a tripod with the letters 'YELH', as reference to the Greek name of the city of Velia, from the nymph's name Yele. The find is part of a group of over a thousand similar specimens referable to the same production, currently the subject of studies $[\mathrm{I}]$ and datable to the second half of the Ist century BC.

To achieve an adequate descriptive quality, the survey was conducted with two different Dino-Lite (<www.dino-lite.eu>) portable digital microscopes, using a calibrated plate -designed by the authors and built for the occasion- to optimize alignment and scaling procedures for the three-dimensional model. The intention, in fact, at this stage of the research, was to increase the descriptive potential of these microscopes.

\section{Materials and methods: 3Dino Plate \& Dino-Lite handheld digital microscopes}

The research activity with the micro-photogrammetric system we called 3Dino was therefore based on the combined use of Dino-Lite portable polarized light microscopes and an original three-dimensional calibrator designed ad hoc. The latter, the 3Dino Plate, consists of a PLA plate obtained with a 3D filament printing, with dimensions of $18 \times 15 \mathrm{~cm}$ and characterized by an orthogonal pattern of 99 truncated conical holes, with a flaring angle of $60^{\circ}$ and diameter of the smaller base equal to $0.35 \mathrm{~mm}$ (fig. I). 


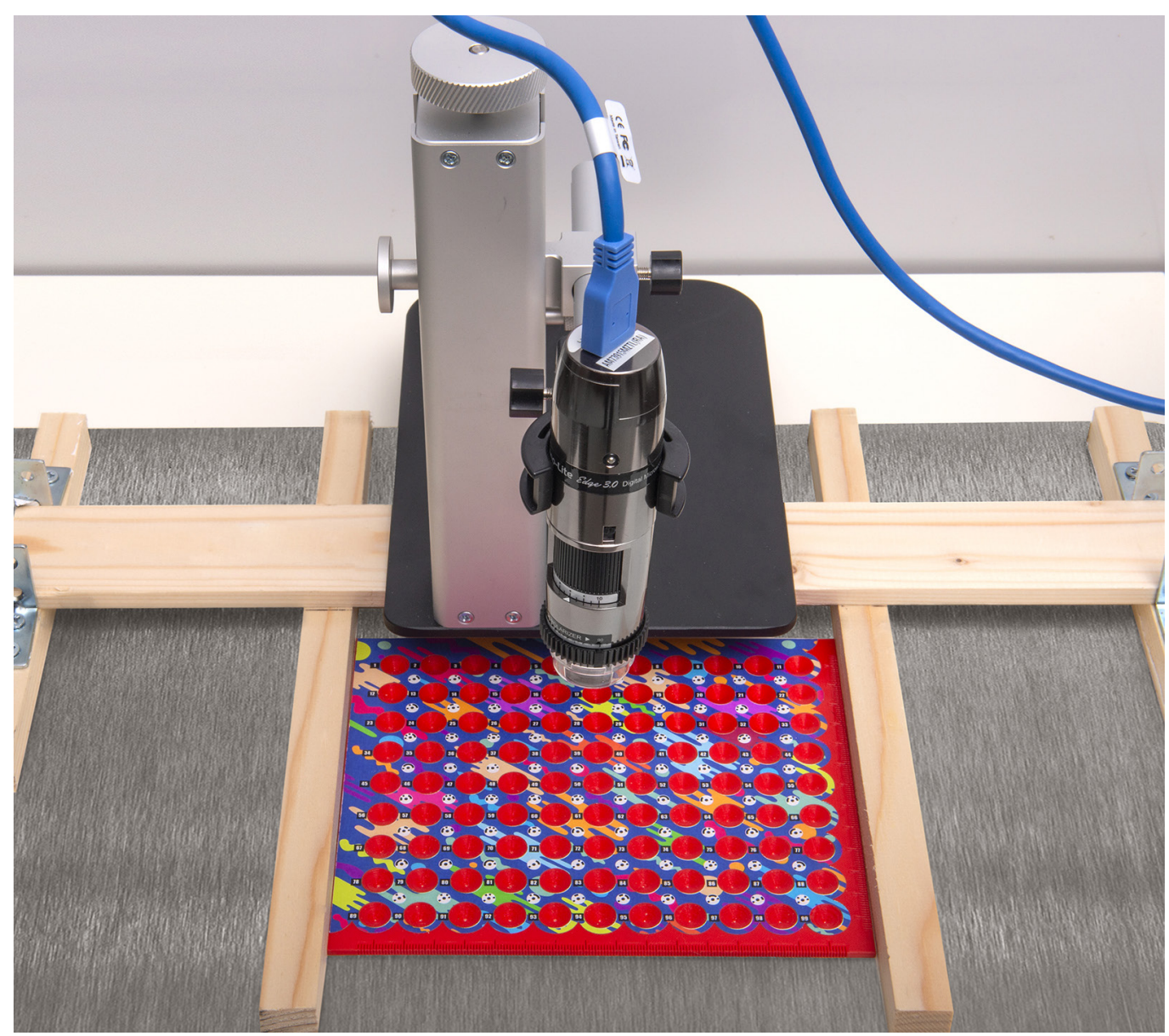

Calibrator's accuracy, based on 3D printing settings [2] and relative errors evaluations, or the actual holes position conformity with respect to project file, has been estimated at 0.1 $\mathrm{mm}$, which can be assumed as system's instrumental error value (i.e., markers accuracy).

Each hole coordinate - with respect to a local reference system- is therefore known to the operator and can be imported into the photogrammetric project: this allows the perforated pattern to be used as a constraint point grid (GCP), homogeneously distributed on the area of interest for the optimization of the parameters governing orientation. In order to guarantee a more robust alignment an adhesive pattern, geometrically and chromatically non-repetitive, equipped with coded targets, was created, and applied to the calibrator's surface.

The system also provided for the housing of the 3Dino Plate on a sliding track, allowing the calibration plate's manual translation with respect to the microscope (fig. 2).

Dino-Lite digital microscopes provide a powerful, portable, and functional solution for detail inspection. The models being tested, the AM4 I I 3ZT Universal [3] and the AM739 I5MZTL High Speed, belong to two different classes, both in terms of technical characteristics and special features (fig. 3), and, consequently, with a quite different price range.

The element that clearly distinguishes the two microscopes is the Working Distance - WD, or the linear distance between the tip of the microscope nozzle and the object to be detected (fig. 4).

The Working Distance directly affects acquired image's magnification (Magnification Factor - MF), framed area (FOV) and the depth of field (DOF). It is trivial to observe that in photogrammetry it is important to establish a fair compromise among these factors, bearing in mind that the further you are from the subject (high WD), the lower the magnification, with a wider field of view and greater depth of field; on the contrary, the closer you are to the 
Fig. 3. Technical specifications of the used Dino-Lite microscope.

\begin{tabular}{|c|c|c|}
\hline Model & AM4113ZT Universal & AM73915MZTL High Speed \\
\hline Dino-Lite range & Dino-Lite Universal & Dino-Lite High Speed \\
\hline Diffuser available & MSAA111A2 (optional accessory) & Yes \\
\hline Polarizer & Yes, linear & Yes, linear \\
\hline Magnification & $10-70 x, 200 x$ & $10-140 x$ \\
\hline Working Distance & Standard & Long \\
\hline Resolution & 1.3 Megapixel (1280x1024) & 5 Megapixel (2592x1944) \\
\hline Maximum Frame rate & 30 fps & 45fps (max 20fps for video recording) \\
\hline Interface & USB 2.0 & USB 3.0 \\
\hline Housing material & Composite/plastic housing & Metal housing \\
\hline & No & Aut. Magn. Reading \\
\hline Special features & & $\begin{array}{c}\text { Extended Dynamic Range (EDR) } \\
\text { Flexible LED Control (FLC) }\end{array}$ \\
\hline Price range & $€ 200,00-€ 350,00$ & $€ 1000,00$ - $€ 1250,00$ \\
\hline
\end{tabular}

subject (low WD), the greater the magnification, with a smaller field of view and less depth. The Working Distance is therefore an extremely important parameter from a practical point of view and. depending on the different microscopes used (fig. 5), inevitably binds the use of different magnifications [4] in the operational phase.

Both models Universal and the High Speed one were combined with the calibrator, fixing the microscope in a special housing, the Dino-Lite RK-I0-EX support, and connecting it to a portable workstation. The acquisitions, therefore, were then carried out with the magnifications summarized in fig. 5, operating with the dedicated DinoCapture 2.0 procedure (<www.dino-lite.eu/index.php/it/software-dino-lite $>$ ) using, for the occasion, also the Extended Dynamic Range - EDR and Extended Depth algorithms Of Field - EDOF, which we will deepen in the next paragraph. The sets of images were thus processed in a SfM software type, Agisoft Metashape, according to the general photogrammetric workflow; the first acquisitions did not consider the variation in focal

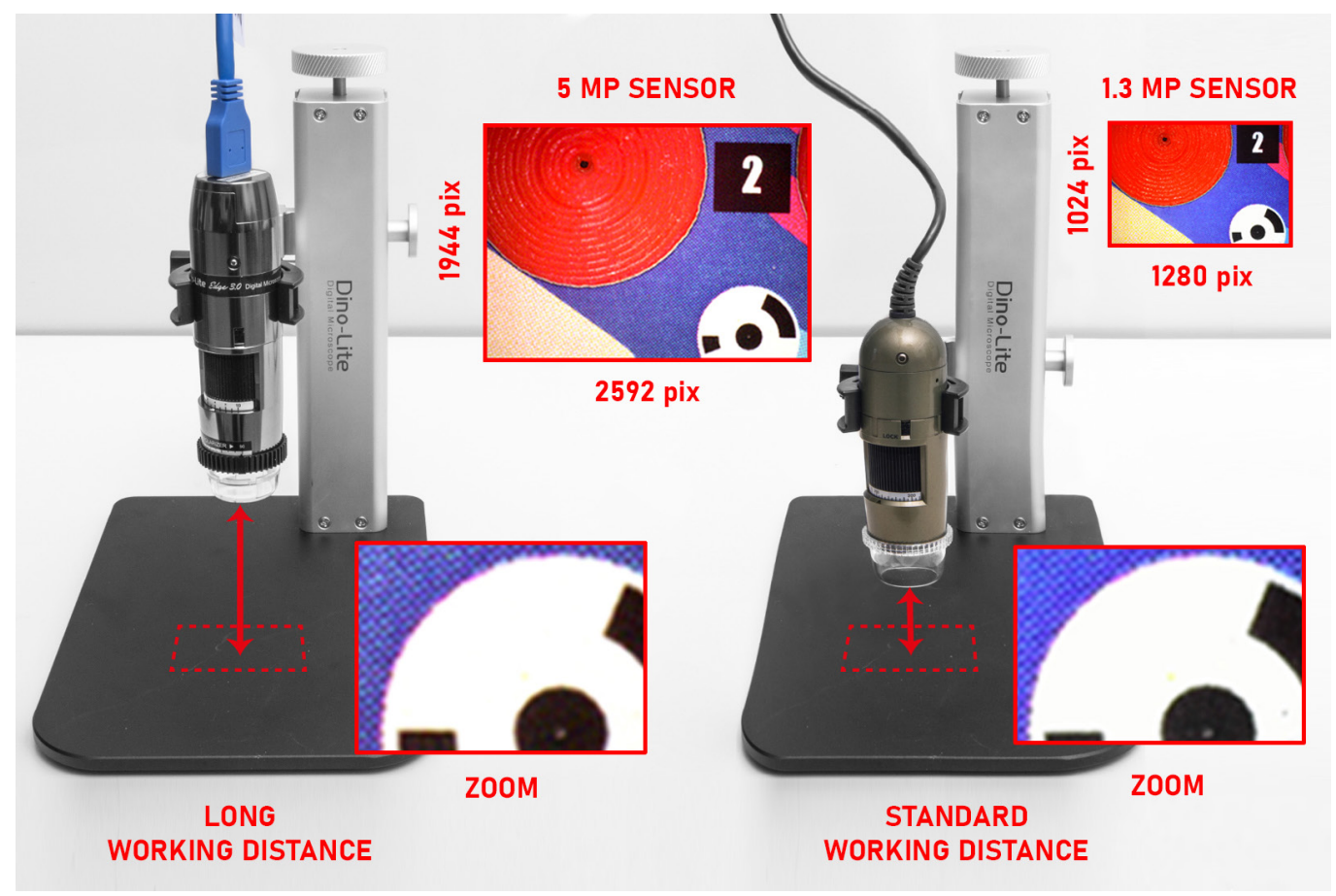


Fig. 5. Based on the magnification ('Magnification Factor'), the values of 'Working Distance', field angle and depth of field have been reported for each microscope; (*) the data does not consider the $15 \mathrm{~mm}$ frontal cap: (*** $^{*}$ (n) from linear interpolation.

\begin{tabular}{|c|c|c|c|c|c|c|}
\hline & \multicolumn{3}{|c|}{ AM4113ZT Universal } & \multicolumn{3}{c|}{ AM73915MZTL High Speed } \\
\hline & $\mathbf{2 0 x}$ & $\mathbf{4 0 x}$ & $\mathbf{5 0 x}$ & $\mathbf{2 0 x}$ & $\mathbf{4 0 x}$ & $\mathbf{9 0 x}$ \\
\hline $\begin{array}{c}\text { Working } \\
\text { Distance* }\end{array}$ & $48,7 \mathrm{~mm}$ & $9,0 \mathrm{~mm}$ & $1,9 \mathrm{~mm}$ & $115,0 \mathrm{~mm}$ & $56,8 \mathrm{~mm}$ & $32,7 \mathrm{~mm}$ \\
\hline $\begin{array}{c}\text { Field Of } \\
\text { View }\end{array}$ & $19,8 \times 14,9 \mathrm{~mm}$ & $9,9 \times 7,4 \mathrm{~mm}$ & $7,9 \times 5,9 \mathrm{~mm}$ & $39,0 \times 29,2 \mathrm{~mm}$ & $9,8 \times 7,3 \mathrm{~mm}$ & $4,3 \times 3,2 \mathrm{~mm}$ \\
\hline $\begin{array}{c}\text { Depth Of } \\
\text { Field }\end{array}$ & $3,6 \mathrm{~mm}$ & $1,4^{* *} \mathrm{~mm}$ & $0,88 \mathrm{~mm}$ & $12,0 \mathrm{~mm}$ & $1,8 \mathrm{~mm}$ & $0,37 \mathrm{~mm}$ \\
\hline
\end{tabular}

distance correlated to the different degrees of magnification (MF), exploiting the new possibilities offered by the SfM software to reconstruct accurate geometries also using less rigorous datasets.

\section{Data Evaluation}

The evaluations of the data generated with the microscopic instrumentation have, first of all, taken into consideration the software management procedures, using Agisoft Metashape, of the micro-photogrammetric images (fig. 6).

Portable digital microscopes, unlike photographic cameras, reveal some specific problems for photogrammetric applications: among the most obvious, and limiting, the lack of Exif data. This information associated with the pixel matrix, as known, retains data relating to camera model, sensor size and focal length. These are essential variables, in the absence of a laboratory calibration, used by any software to process the appropriate calculations for distortion estimation and camera location. To this lack of information must be added the limited depth of field of these instruments: the possibility offered by the Extended Depth Of Field - EDOF to overcome this limit by acquiring and combining more shots on different focus planes, was not an improvement in terms of terms of image quality, but above all not very suitable and decisive for the case study (with this method it is also not possible to estimate a unique value of the focal distance for the image). The other tested mode, Extended Dynamic Range - EDR, while not offering a solution to the reduced depth of field, by superimposing images with different exposures, proved to be useful for characterizing details of darker or brighter areas, highlighting a particular effectiveness for reflective surfaces, although the used microscopes already benefited from polarized light.

It should be borne in mind that the two modes, Extended Depth Of Field - EDOF and Extended Dynamic Range - EDR, require a longer acquisition time (about 20 seconds for each single shot compared to Normal mode), due to the pre-processing of the data carried out directly in the proprietary application DinoCapture 2.0 (fig. 7).

Right after a quick analysis it was possible to evaluate the results obtained by the Universal model as appreciable (fig. 8), which guaranteed outputs comparable to the High Speed model, despite being considered entry-level. From our tests and graphs referred to in the previous tables and images, the study of a slice of a point cloud exported from the four homologous 3D models is particularly exemplary: although it is not possible to estimate

\begin{tabular}{|c|c|c|c|c|c|c|c|}
\hline Model & Mode & $\begin{array}{c}\text { Aligned } \\
\text { Cameras }\end{array}$ & $\begin{array}{c}\text { Ground } \\
\text { resolution } \\
\text { (GSD) }\end{array}$ & $\begin{array}{c}\text { Tie } \\
\text { Points** }\end{array}$ & Projections & $\begin{array}{c}\text { RMS } \\
\text { reprojection } \\
\text { error }\end{array}$ & $\begin{array}{c}\text { Control } \\
\text { Points } \\
\text { Error*** }\end{array}$ \\
\hline $\begin{array}{c}\text { AM4113ZT } \\
\text { Universal }\end{array}$ & Normal & $110 / 111$ & $4,01 \mu \mathrm{m} / \mathrm{pix}$ & 145.613 & 411.610 & 1,47 pix & $0,076 \mathrm{~mm}$ \\
\hline & EDOF & $134 / 135$ & $6,49 \mu \mathrm{m} / \mathrm{pix}$ & 104.520 & 266.653 & 1,01 pix & $0,094 \mathrm{~mm}$ \\
\hline $\begin{array}{c}\text { AM73915MZTL } \\
\text { High Speed }\end{array}$ & EDR & $100 / 103$ & $3,45 \mu \mathrm{m} /$ pix & 171.702 & 471.944 & 0,77 pix & $0,094 \mathrm{~mm}$ \\
\cline { 2 - 9 } & Normal & $148 / 152$ & $4,44 \mu \mathrm{m} / \mathrm{pix}$ & 169.503 & 455.609 & 1,77 pix & $0,089 \mathrm{~mm}$ \\
\hline
\end{tabular}


Fig. 7. time comparison of DinoCapture 2.0 acquisition and Agisoft Metashape processing The processing was performed with the same graphic workstation equipped with Intel 19 9900k CPU, RTX 2080Ti GPU, and 64GB of RAM.

\begin{tabular}{|c|c|c|c|c|c|c|c|c|c|}
\hline Model & $\begin{array}{c}\text { Operative } \\
\text { Mode }\end{array}$ & Acquisition & Alignment & $\begin{array}{c}\text { Marker } \\
\text { positioning }\end{array}$ & $\begin{array}{l}\text { Dense } \\
\text { Point } \\
\text { Cloud }\end{array}$ & Model & DSM & Ortho & TOT \\
\hline $\begin{array}{c}\text { AM4113ZT } \\
\text { Universal }\end{array}$ & Normal & $15^{\prime}$ & $1^{\circ} 08^{\prime}$ & $30^{\prime}$ & 17 & $30^{\prime}$ & 8" & $46^{\prime \prime}$ & $2^{\circ} 41^{\prime}$ \\
\hline \multirow{3}{*}{$\begin{array}{c}\text { AM73915MZTL } \\
\text { High Speed }\end{array}$} & $\begin{array}{c}\text { Extended } \\
\text { Depth Of } \\
\text { Field } \\
\text { EDOF }\end{array}$ & $45^{\prime}$ & $1^{\circ} 56^{\prime}$ & $30^{\prime}$ & 9' & $40^{\prime}$ & $11 "$ & $1^{\prime}$ & $4^{\circ} 02^{\prime}$ \\
\hline & $\begin{array}{c}\text { Extended } \\
\text { Dynamic } \\
\text { Range } \\
\text { EDR }\end{array}$ & $45^{\prime}$ & 58 & $30^{\prime}$ & $11^{\prime}$ & $18^{\prime}$ & $11 "$ & $35 "$ & $2^{\circ} 43^{\prime}$ \\
\hline & Normal & $15^{\prime}$ & $2^{\circ} 13^{\prime}$ & $30^{\prime}$ & $11^{\prime}$ & $1^{\circ} 02^{\prime}$ & $11 "$ & $1 '$ & $4^{\circ} 12^{\prime}$ \\
\hline
\end{tabular}

the uncertainties in an absolute way (at the moment it has not yet been brought to term a range-based survey to be taken as reference model) the detail of the cloud produced with the data of the Universal model is however evident (figs. 9, I0). Despite the confirmations about the performance of tested instrumentation, the data in possession do not allow, for now, more thorough, and analytical evaluations on the system's accuracy, which will make it necessary to carry out new studies to track back the parametric variables of its internal orientation.

Further investigations will be conducted by comparing the SfM procedures results with other survey technologies for the assumption of a reference surface to be used as an 'absolute' model of comparison.

\section{Considerations and future developments}

The tests performed are suitable for defining the use of portable microscopes for photogrammetric purposes as valid. The actual resolution of the data, the quality / price ratio, portability, and relative ease of use of these instruments, undoubtedly represent the strength of the method. On the other hand, it must be specified that, in order to pursue the primary objective of each survey, namely the verifiability and reproducibility of the metric value, some key aspects still need to be better defined.

\begin{tabular}{|c|c|c|c|c|c|c|c|c|}
\hline \multirow{2}{*}{ Model } & \multirow{2}{*}{ Mode } & \multicolumn{2}{|c|}{ Dense Point Cloud } & \multicolumn{2}{|c|}{ Model } & \multirow{2}{*}{$\begin{array}{c}\text { DEM } \\
\begin{array}{c}\text { Elevation } \\
\text { Range }\end{array}\end{array}$} & \multicolumn{2}{|c|}{ Ortho } \\
\hline & & Points & Faces & Vertices & $\begin{array}{c}\text { Point } \\
\text { Density }\end{array}$ & & Size & $\begin{array}{l}\text { Image } \\
\text { Weight }\end{array}$ \\
\hline 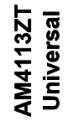 & Normal & 32.148 .558 & 6.429 .711 & 3.222 .831 & $\begin{array}{c}6,23 \times 10^{4} \\
\text { points } / \mathrm{mm}^{2}\end{array}$ & $3,0 \mathrm{~mm}$ & $\begin{array}{c}3850 \times 3893 \\
\text { pix }\end{array}$ & $\begin{array}{l}60,1 \\
M B\end{array}$ \\
\hline \multirow{3}{*}{ 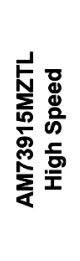 } & EDOF & 49.018 .983 & 9.803 .616 & 4.949 .755 & $\begin{array}{c}6,49 \times 10^{4} \\
\text { points } / \mathrm{mm}^{2}\end{array}$ & $7,5 \mathrm{~mm}$ & $\begin{array}{c}6026 \times 5848 \\
\text { pix }\end{array}$ & $\begin{array}{l}85,2 \\
M B\end{array}$ \\
\hline & EDR & 24.577 .548 & 4.915 .506 & 2.465 .745 & $\begin{array}{c}8,41 \times 10^{4} \\
\text { points } / \mathrm{mm}^{2}\end{array}$ & $2,8 \mathrm{~mm}$ & $\begin{array}{c}6043 \times 5879 \\
\text { pix }\end{array}$ & 77,8 \\
\hline & Normal & 48.697 .228 & 9.739 .215 & 4.911 .372 & $\begin{array}{c}5,08 \times 10^{4} \\
\text { points } / \mathrm{mm}^{2}\end{array}$ & $6,0 \mathrm{~mm}$ & $\begin{array}{c}6265 \times 6108 \\
\text { pix }\end{array}$ & $\begin{array}{c}106,0 \\
M B\end{array}$ \\
\hline
\end{tabular}


Fig. 9. Comparison of models generated with different datasets: the first acquired with the 'AM4I I3ZT Universa' microscope in Norma mode (i.e., in the absence of 'special features'): the second third and fourth models related to the models related to the acquisitions obtained with the 'AMTJYI5MZT High Speed microscope respectively in EDOF, EDR and Normal mode. The 'White Balance' has been set for an automatic colour analysis, without images post-corrections, resulting, however, a clear difference in the RGB data generated with the 'AM4II3ZT

Universal' model (tending to green, top left)

compared to those of the 'AM73915MZTL High Speed' model (more chromatically accurate)
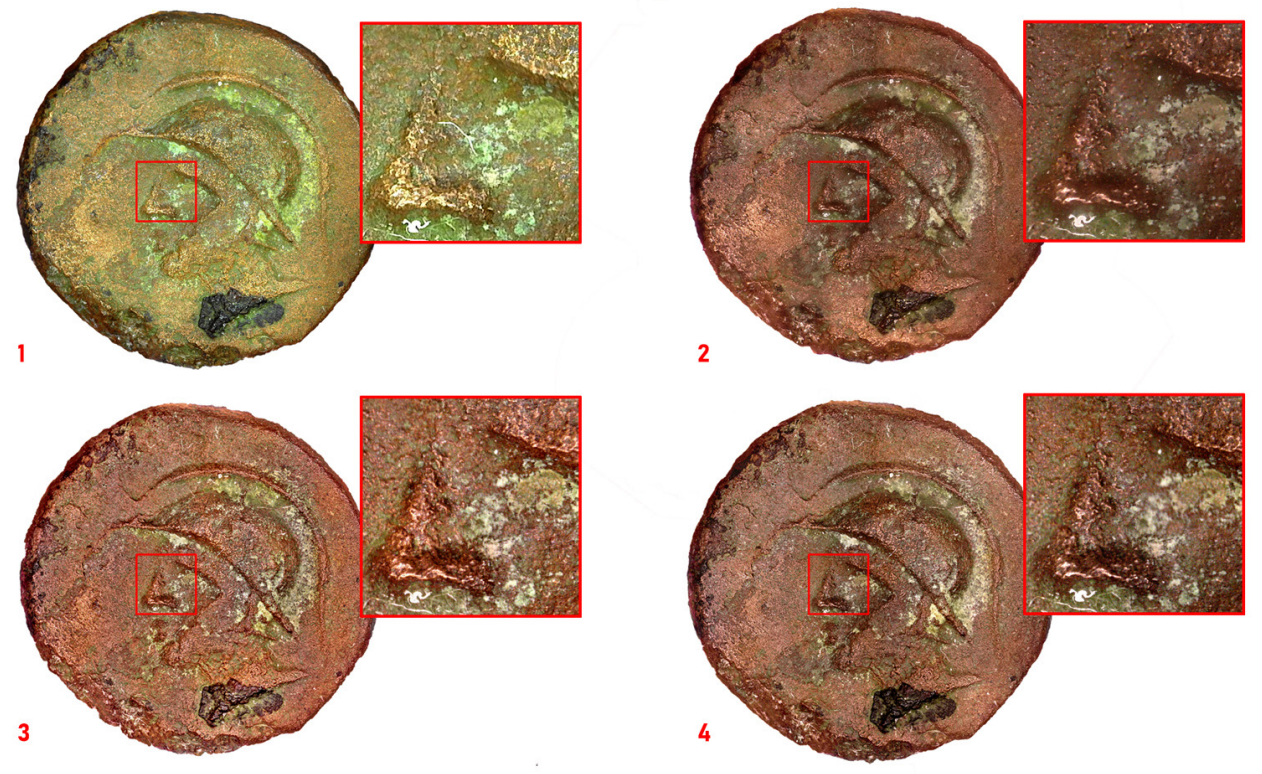

In addition to software modifications, allowing Exif data to be saved, some modifications necessary to the calibrator in fig. I are now clear. First, the thickness of the 3Dino Plate, which must be smaller than the one proposed. This will allow the reduction of the distance between markers, allowing the identification of multiple points of known coordinates and therefore to have, in addition to Control Points, Check Points too. Furthermore, it was observed the opportunity to modify plate's geometry, from rectangular to round, to adapt it to a motorized rotating plate, to facilitate possible acquisitions with an inclined axis, as well as providing it with protrusions on several levels, and to offer more planes of focus and a more rigorous tool for evaluating depths. In addition, the acquisition procedure will have to be reconsidered in terms of magnification, blocking the microscope ring allowing to modify the Magnification Factor -keeping, therefore fixed the focal distance- and approaching and/or moving away from the object, or changing the relative Working Distance,

Fig. 10. On the left, comparison among point clouds sections generated with different microscope models: the first with 'AM4I I 3ZT Universal' in Normal mode (i.e., in the absence of 'special features'); the second, third and fourth with the 'AM739I5MZTL High Speed' microscope, respectively in EDOF, EDR and Normal mode. On the right the point cloud obtained with the 'Universt' microscope with the positioscope with the positioning of the slice chosen for the
above comparison.
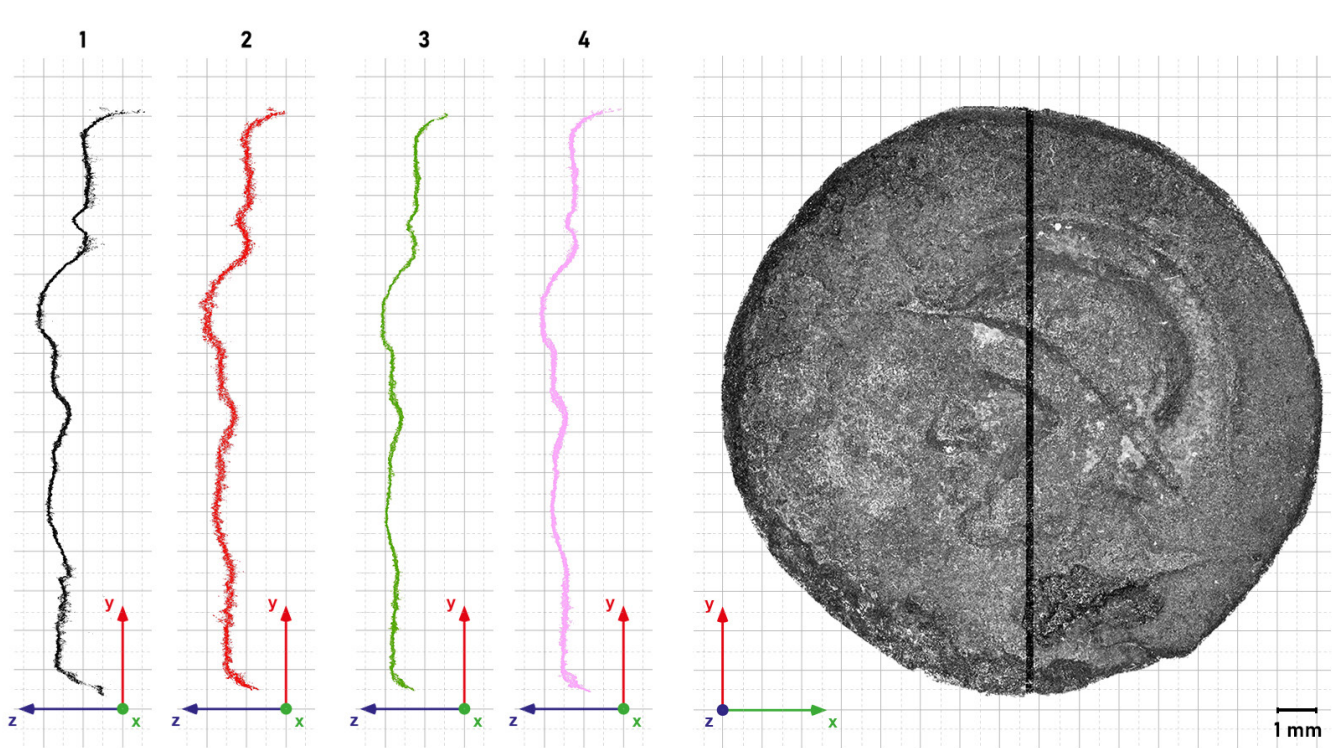
Fig. II. Global and relative deviations on the individual axes, expressed in millimetres. The data coming from the 'Universal' microscope were wer a deviations of he survey deviations of the survey performed with the 'High Speed microscope (In Normal, EDOF or EDR mode).

Fig. 12. Comparison among Dense Surface Models generated with different datasets: the first with the 'AM4I I $3 Z \mathrm{~T}$ Univith ' 'AM MIII in Normal micro in Normal mode (i.e., in the absence of special features'); the second, third and fourth models with the AM739I5MZTL High Speed' microscope respectively in EDOF, EDR and Normal mode.
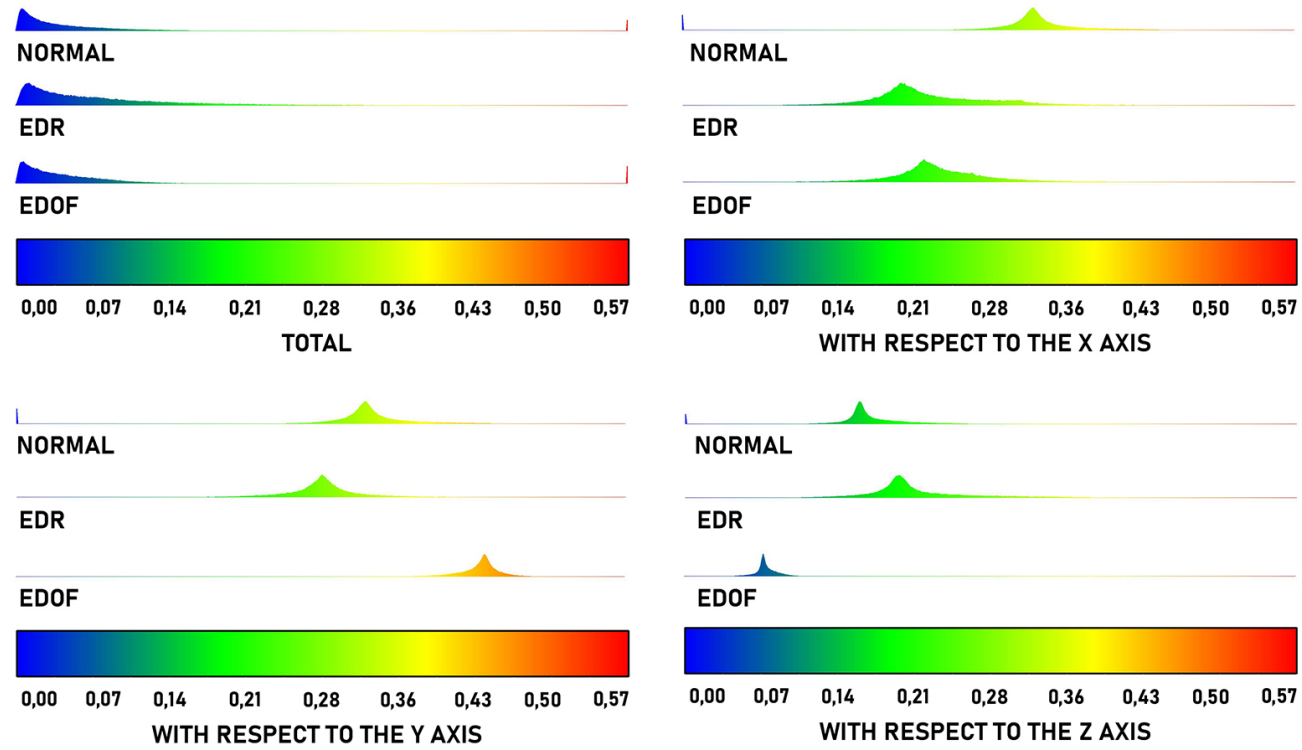

always ensuring sufficient overlap (i.e., operating differently from combining shots with different MFs and WDs).

Despite the problems, within the framework of modern micro-technologies, more interesting ideas prevail from this work in progress for the three-dimensional documentation of very small artifacts that would otherwise be difficult to represent.
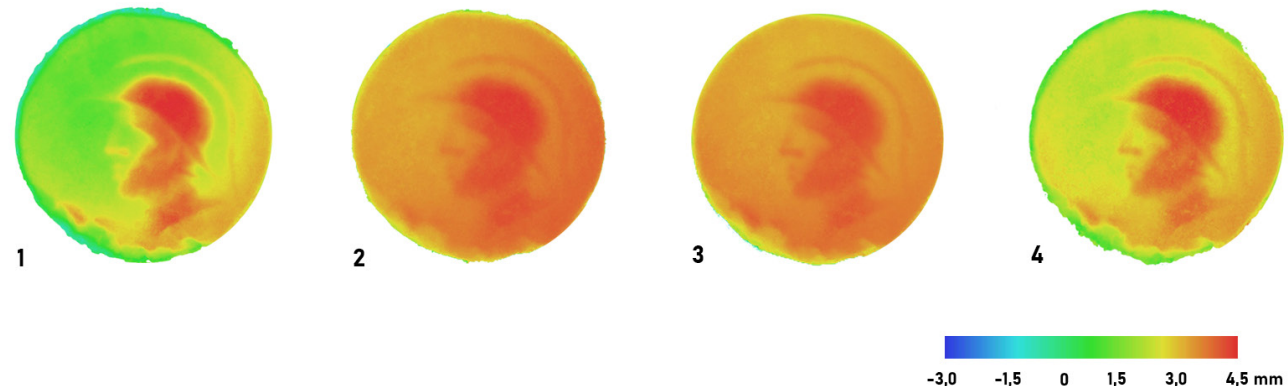

\section{Acknowledgments}

We sincerely thank the IDCP Digital Innovation -Jan Boers, Danielle van Duijvendijk and Ivo Manders- and, of course, the Dino-Lite Digital Microscope for the support and equipment made available for the research.

We also thank Giacomo Pardini of the Department of Cultural Heritage Sciences of the University of Salerno for granting access to the numismatic archive and for supporting the publication of these results.

\section{Notes}

[I] The issue of Velia 'Atena/Tripode' is being studied by Federico Carbone, as part of the research project on monetary finds from Velia entitled L a moneta di basso conto a Elea/Velia: uso e produzione (Directed by Renata Cantilena) and the Art Bonus project Le monete di Elea/Velia - Un restauro per la conoscenza e la valorizzazione del patrimonio archeologico della cittc̀ di Parmenide, carried out in collaboration with Soprintendenza Archeologia, Belle Arti e Paesaggio per le province di Salerno e Avellino and supported by the Fondazione Nazionale delle Comunicazioni (Rome)

[2] Below are the settings of the 3D printer Creality CR- 10 v 1.0 for the realization of the 3Dino Plate: bed temperature $50^{\circ}$ C; nozzle temperature $200^{\circ} \mathrm{C}$; nozzle diameter $0.3 \mathrm{~mm}$; material used PLA $1.75 \mathrm{~mm}+/-0.05 \mathrm{~mm}$; layer height $0.12 \mathrm{~mm}$; infill $20 \%$; infill pattern cubic; printing speed $50 \mathrm{~mm} / \mathrm{s}$. The time taken was about 13 hours. The intrinsic characteristics of PLA make prints made with this material subject to relatively rapid deterioration due to moisture absorption and sensitivity to UV rays; this leads the authors to consider the choice of this material only valid for plate's prototyping phase. A steel prototype of the 3 Dino Plate is being produced with the EOSINT M270 Xtended version printer 
[3] The AM4I I 3ZT Universal model was the subject of the previous publication, of which this contribution represents the natural continuation [Antinozzi, Ronchi, Barba 2020].

[4]The aim of obtaining a greater detail leads to the use of high magnifications, but the different Working Distance of the two microscopes has not always allowed the use of the same magnification values. For example, magnification values close to 50x would have made contact of the AM4 I I 3ZT Universal microscope with the coin surface.

\section{References}

Adamopoulos E., Rinaudo F. (2019). An Updated Comparison on Contemporary Approaches for Digitization of Heritage Objects. In Catelani M., Daponte P. (a cura di). Atti di 2019 IMEKO TC-4 International Conference on Metrology for Archaeology and Cultural Heritage. Firenze, Italia, 4-6 Dicembre, 2019, pp. I-6.

Antinozzi S., Ronchi D., Barba S. (2020). Macro e micro fotogrammetria per la virtualizzazione della laminetta orfica (V-IV a.C.) del Museo Nazionale di Vibo Valentia/Macro and Micro Photogrammetry for the Virtualization of the Orphic Foil (V-IV B.C) of National Museum of Vibo Valentia. In Arena A. et al. (a cura di). Connettere. Un disegno per annodare e tessere. Atti del $42^{\circ}$ Convegno Internazionale dei Docenti delle Discipline della Rappresentazione / Connecting. Drawing for weaving relationships. Proceedings of the 42 nd International Conference of Representation Disciplines Teachers. Milano: Franco Angeli, pp. I 538 - I 555.

Apollonio F.l., Fantini F., Garagnani S., Gaiani M.A. (202I). Photogrammetry-Based Workflow for the Accurate 3D Construction and Visualization of Museums Assets. In Remote Sensing, vol. I3, Issue 3, 486, pp. I-40.

Bolognesi C. M., Fiorillo F. (2018). Optimization of texture mapping process in the Reality-Based Modeling application. In Salerno R. (a cura di). Rappresentazione / Materiale / Immateriale. Atti del $40^{\circ}$ Convegno internazionale dei Docenti delle Discipline della Rappresentazione. Milano, Italia, I5-I7 settembre, 20 I8, pp. 337-342.

Esmaeili F., Ebadi H. (2017). Handy Microscopic Close-Range Videogrammetry. In The International Archives of the Photogrammetry, Remote Sensing and Spatial Information Sciences, vol. XLII-4M4, pp. 65-67.

Galantucci L.M., Pesce M., Lavecchia F. (2016). A powerful scanning methodology for 3D measurements of small parts with complex surfaces and sub millimeter-sized features, based on close range photogrammetry. In Precision Engineering, vol. 43, pp. $211-219$.

Gontard L. C., Schierholz R., Yu S., Cintas J., Dunin-Borkowski R. E. (20 I 6). Photogrammetry of the three-dimensional shape and texture of a nanoscale particle using scanning electron microscopy and free software. In Ultramicroscopy, vol. I69, pp. 80-88.

Hansen H.N., Carneiro K., Haitjema H., De Chiffre L. (2006). Dimensional Micro and Nano Metrology. In CIRP Annals, vol. 55 , Issue 2, pp. $721-743$

Hess M., MacDonald L.W., Valach J. (20I8). Application of multi-modal 2D and 3D imaging and analytical techniques to document and examine coins on the example of two Roman silver denarii. In Heritage Science, vol. 6, Issue 5, pp. I-22.

Mitchell H. L., Kniest H.T. (1999). Digital Photogrammetry and Microscope Photographs. In Photogrammetric Record, n. I 6, pp. 695-704.

Morena S., Barba S., Álvaro-Tordesillas A. (2019). Shining 3D Einscan-Pro, application and validation in the field of Cultural Heritage, from the Chillida-Leku Museum to the Archaeological Museum of Sarno. In The International Archives of the Photogrammetry, Remote Sensing and Spatial Information Sciences, vol. XLII-2/W I 8, pp. I 35- I 42.

Plisson H., Zotkina L.V. (20I5). From 2D to 3D at macro- and microscopic scale in rock art studies. In Digital Applications in Archaeology and Cultural Heritage, vol. 2, Issues 2-3, pp. I02-1 19.

Pieraccini M., Guidi G., Atzeni C. (200I). 3D digitizing of cultural heritage. In Journal of Cultural Heritage, n. 2, pp. 63-70.

Tolksdorf J. F., Elburg R., Reuter T. (2017). Can 3D scanning of countermarks on Roman coins help to reconstruct the movement of Varus and his legions. In Journal of Archaeological Science: Reports, vol. II, pp. 400-4I 0.

\section{Authors}

Sara Antinozzi, Università degli Studi di Salerno, santinozzi@unisa.it

Diego Ronchi, Università degli Studi di Salerno, dronchi@unisa.it

Salvatore Barba, Università degli Studi di Salerno, sbarba@unisa.it

To cite this chapter. Antinozzi Sara, Ronchi Diego, Barba Salvatore (2021). 3Dino System, come accorciare le distanze nei rilievi di precisione/3Dino System, Shortening Distances in Precision Surveys. In Arena A. Arena M. Mediati D. Raffa P. (a cura di). Connettere. Un disegno per annodare e tessere Linguagai Distanze Tecnologie. Atti del $42^{\circ}$ Convegno Internazionale dei Docenti delle Discipline della Rappresentazione/Connecting Drawing for Milano: FrancoAngeli, pp. 1922-1941. 